\title{
The metal-rich halo tail extended in $|z|$ : a characterization with Gaia DR2 and APOGEE
}

\author{
Emma Fernández-Alvar ${ }^{1,2 \star}$, José G. Fernández-Trincado ${ }^{3,4} \dagger$, Edmundo Moreno ${ }^{2}$,
} William J. Schuster ${ }^{5}$, Leticia Carigi ${ }^{2}$, Alejandra Recio-Blanco ${ }^{1}$, Timothy C. Beers ${ }^{6}$, Cristina Chiappini ${ }^{7,9}$, Friedrich Anders ${ }^{7,9}$, Basílio X. Santiago ${ }^{8,9}$, Anna B. A. Queiroz ${ }^{8,9}$, Angeles Pérez-Villegas ${ }^{10}$, Olga Zamora ${ }^{11}$, D. A. García-Hernández ${ }^{11}$ and Mario Ortigoza-Urdaneta ${ }^{3}$

${ }^{1}$ Université Cote d'Azur, Observatoire de la Cote d'Azur, CNRS, 06300, Laboratoire Lagrange

${ }^{2}$ Instituto de Astronomía, Universidad Nacional Autónoma de México, AP 70-264, 04510, Ciudad de México, México

${ }^{3}$ Instituto de Astronomía y Ciencias Planetarias, Universidad de Atacama, Copayapu 485, Copiapó, Chile

${ }^{4}$ Institut Utinam, CNRS UMR6213, Univ. Bourgogne Franche-Comté, OSU THETA, Observatoire de Besançon, BP 1615, 25010 Besançon Cedex, France

${ }^{5}$ Instituto de Astronomía, Universidad Nacional Autónoma de México, AP 106, 22800 Ensenada, B. C., México

${ }^{6}$ Department of Physics and JINA Center for the Evolution of the Element (JINA-CEE), University of Notre Dame,

Notre Dame, IN 46556 USA

${ }^{7}$ Leibniz-Institut fur Astrophysik Potsdam (AIP), An der Sternwarte 16, 14482 Potsdam, Germany

${ }^{8}$ Instituto de Física, Universidade Federal do Rio Grande do Sul, Caixa Postal 15051, 91501-970 Porto Alegre, Brazil

${ }^{9}$ Laboratório Interinstitucional de e-Astronomia-LIneA, Rua Gal. José Cristino 77, 20921-400 Rio de Janeiro, Brazil

${ }^{10}$ Universidade de São Paulo, IAG, Rua do Matão 1226, Cidade Universitária, 05508-900, São Paulo, Brazil

${ }^{11}$ Instituto de Astrofísica de Canarias, 38205 La Laguna, Tenerife, Spain

Accepted XXX. Received YYY; in original form ZZZ

\begin{abstract}
We report an analysis of the metal-rich tail $([\mathrm{Fe} / \mathrm{H}]>-0.75)$ of stars located at distances from the Galactic plane up to $|z| \sim 10 \mathrm{kpc}$, observed by the Apache Point Observatory Galactic Evolution Experiment (APOGEE). We examine the chemistry, kinematics, and dynamics of this metal-rich sample using chemical abundances and radial velocities provided by the fourteenth APOGEE data release (DR14) and proper motions from the second Gaia data release (DR2). The analysis reveals three chemically different stellar populations in the $[\mathrm{Mg} / \mathrm{Fe}]$ vs. $[\mathrm{Fe} / \mathrm{H}]$ space - a high- $[\mathrm{Mg} / \mathrm{Fe}]$ and low- $[\mathrm{Mg} / \mathrm{Fe}]$ populations, and a third group with intermediate $[\mathrm{Mg} / \mathrm{Fe}] \sim+0.1-$ as well as for other chemical elements. We find that they are also kinematically and dynamically distinct. The high- $[\mathrm{Mg} / \mathrm{Fe}]$ population exhibits a prograde rotation which decreases down to 0 as $\left|z_{\max }\right|$ increases, as well as eccentric orbits that are more bound and closer to the plane. The low- $[\mathrm{Mg} / \mathrm{Fe}]$ stars are likely Sagittarius members, moving in less-bound orbits reaching larger distances from the centre and the Galactic plane. The intermediate-Mg stars resembles the two stellar overdensities lying about $|z| \sim 5$ kpc recently reported in the literature, for which a disc origin has been claimed. We report the identification of new members of these two disc-heated overdensities.
\end{abstract}

Key words: Galaxy - halo - stellar populations

* E-mail: emmafalvar@gmail.com

$\dagger$ E-mail: jfernandez@obs-besancon.fr

\section{INTRODUCTION}

The bulk of the stars in the Galactic halo are characterized by low metallicities, indicating that they were born during the first stages of Galactic formation, from gas enriched by the first generations of stars.

The seminal monolythic collapse model of Eggen et al. 
(1962) predicted that halo stars are the first stars born during the collapse of the protogalactic cloud, before the gas settled and formed a disc. However, within the current cosmological paradigm, the $\Lambda$ Cold Dark Matter model describes galaxy formation as resulting from the assembly of larger structures from mergers of smaller substructures. This implies that the Galactic halo was populated by stars born in satellite galaxies which underwent different, individual starformation histories, and have been accreted into the Milky Way over time. Observational evidence gathered during the last decades provide support to this model, with the detection of stellar density substructures accross the Galactic halo (e.g., Grillmair 2009, Newberg \& Carlin 2015, Bernard et al. 2016).

Recent studies of the Galactic stellar halo have provided signatures for a variation in the spatial, chemical, and kinematical properties of halo stars with distance from the Galactic Centre - a break in the spatial distribution (Deason, Belokurov \& Evans 2011; Ablimit \& Zhao 2018); a gradient in the mean iron abundance (which is usually considered as a metallicity indicator in stars) with distance (Fernández-Alvar et al. 2015) correlated with the mean rotational velocity component (Carollo et al. 2007, 2010; Beers et al. 2012; see also the discussions in Schönrich et al. 2014 and An et al. 2015); and signatures of different chemical enrichment with distance (Fernández-Alvar et al. 2015, 2017; Lee et al. 2017).

Finally, two chemically distinct stellar populations in terms of the $\alpha$-to-iron enrichment (Nissen \& Schuster 2010, 2011; Hawkins et al. 2015; Bergemann et al. 2017; Hayes et al. 2018a; Fernández-Alvar et al. 2018) were also detected. All of these observational results led to the claim for a possible dual formation scenario for the Galactic halo, comprising in-situ star formation as well as accretion from satellite galaxies. Several numerical galaxy formation studies have considered the relative contribution of in-situ star formation with respect to an accreted origin throughout the halo (e.g., Font et al. 2011; Tissera et al. 2012, 2013, 2014), and found that this relative fraction affects the global chemical and kinematical properties of the stars and their variation with distance.

Although the majority of the halo stars have $[\mathrm{Fe} / \mathrm{H}]$ $<-1.0$, some of the previously mentioned works revealed stars at higher metallicities that strongly differ in kinematics with respect to disc stars, and instead exhibit halo-like motions (Nissen \& Schuster et al. 2010, 2011; Schuster et al. 2012; Fernández-Alvar et al. 2018). It is in this metal-rich range, $-1.6<[\mathrm{Fe} / \mathrm{H}]<-0.4$, where the two distinct $[\alpha / \mathrm{Fe}]$ populations were detected in the first place.

Based on the kinematical and dynamical properties of these metal-rich halo stars, and their overlap with the thickdisc chemical patterns in the $[\alpha / \mathrm{Fe}]$ vs. $[\mathrm{Fe} / \mathrm{H}]$ space, Nissen \& Schuster $(2010,2011)$ and Schuster et al. (2012) proposed that the high- $\alpha$ stars could be old disc or bulge stars heated to halo kinematics by merging satellite galaxies, or could have formed during the collapse of a proto-Galactic gas cloud. Conversely, from their kinematics, the low- $\alpha$ populations would have been accreted, presumably from dwarf galaxies with low star-formation rates.

Bonaca et al. (2017) kinematically identified halo stars within $3 \mathrm{kpc}$ of the Sun moving with relative speeds larger than $220 \mathrm{kms}^{-1}$ with respect to the Local Standard of Rest
(LSR). Surprisingly, half of this kinematically-defined halo sample have $[\mathrm{Fe} / \mathrm{H}]>-1.0$. Because these stars move on orbits that are preferentially aligned with the disc rotation, they proposed that these metal-rich halo stars may have formed in-situ in the disc, and later were perturbed by interactions with a more massive dwarf galaxy, rather than being accreted from satellite galaxies. These authors made use of the Gaia DR1 (Gaia Collaboration et al. 2016) and the Tycho-Gaia solution (Michalik, D., Lindegren, L., \& Hobbs 2015) to derive parallaxes and proper motions, which lacked the greater accuracy achieved in Gaia DR2.

Taking advantage of the enourmous improvement in accuracy and precision provided by the Gaia DR2 data (Gaia Collaboration et al. 2018), Helmi et al (2018) claimed an accretion origin for stars with halo-like motions in the Solar Neighbourhood, which they associated with a relic of what could be the last major merger event to have occurred in the Galaxy, "Gaia-Enceladus". Previously, Belokurov et al. (2018) had detected a radial anisotropy in halo stars at $[\mathrm{Fe} / \mathrm{H}]>-1.7$, suggesting that most of the inner, metal-rich halo would be dominated by stars accreted from a single massive merger event. This idea was later corroborated by several works (Myeong et al. 2018a,b, Deason et al. 2018, Lancaster et al. 2018, Simion et al. 2019).

Haywood et al. (2018) also supported this idea, from their analysis of two overdensities in the HertzsprungRussell diagram (HRD) revealed by Gaia Collaboration et al. (2018b), atributted to the halo and the disc. They compared the two Nissen \& Schuster populations, also using results from Gaia DR2, and found that these two groups of stars are located over the two different sequences in the $\mathrm{HRD}$, providing evidence that they are in fact two different stellar populations (the situation remained unclear for stars with $[\mathrm{Fe} / \mathrm{H}]<-1.0)$. They also analysed the kinematical and dynamical properties of the two groups considering stars from the Apache Point Observatory Galactic Evolution Experiment fourteenth data release (APOGEE DR14; Majewski et al. 2017, Abolfathi et al. 2018), confirming differences between them, and argued for a likely disc origin for the high- $\alpha$, metal-rich stars, and an accreted origin of the low- $\alpha$ group and metal-poor stars, commensurate with the Helmi et al (2018) results.

Recently, Mackereth et al. (2018) provided yet more evidence from a detailed examination of the metal-poor stars in the APOGEE DR14 database. They found that $2 / 3$ of nearby halo stars are characterized by orbital eccentricities larger than 0.8 , and chemical trends similar to current massive dwarf galaxy satellites of the Milky Way. This group of stars would presumably belong to the same accretion event. They also exhibit slightly retrograde motions, and reach larger heights from the plane and apocentric distances during their orbits. Interestingly, they presented the same radial anisotropy as found by Belokurov et al. (2018) . A comparison with the EAGLE suite of cosmological simulations constrained the mass of such an accreted satellite to the range $10^{8.5} \lesssim M_{*} \lesssim 10^{9} M_{\odot}$.

In this work we aim to analyse the metal-rich halo component at distances beyond the Solar Neighbourhood. For this purpose we consider the chemical, kinematical, and dynamical properties of a halo sample selected from APOGEE DR14 combined with Gaia DR2 (Gaia Collaboration et al. 2016, 2018b). APOGEE is one of the Sloan Digital Sky Sur- 
vey IV programs (Blanton et al. 2017) devoted to exploration of the various Galactic components, in order to understand the formation and evolution of our Galaxy.

This paper is organized as follows. The APOGEE data and our criteria to select halo stars are briefly explained in Section 2. In Section 3 we describe the chemical, kinematical, and dynamical analyses performed and the results obtained. Section 4 is a discussion of the implications of our results. Finally, we summarize our main conclusions in Section 5.

\section{DATA}

We select our sample of halo stars from the APOGEE fourteenth data release (DR14; Abolfathi et al. 2018). This data release includes data from the SDSS-III (Eisenstein et al. 2011) APOGEE-1 program (which comprises observations gathered from August 2011 to July 2014 - see Zasowski et al. 2013), as well as from the first two years of SDSS-IV APOGEE-2 data (July 2014 - July 2016 - see Zasowski et all. 2017). Observations were taken using the Sloan Foundation 2.5-meter telescope (Gunn et al. 2006) at Apache Point observatory, and the APOGEE spectrograph (Wilson et al. 2010). Data for both programs was processed (Nidever et al. 2015), and chemical abundances and radial velocities derived (Holtzman et al. 2015), with the APOGEE Stellar Spectra Parameter Pipeline (ASPCAP; García-Pérez et al. 2016).

We choose field stars located at distances from the Galactic plane larger than $5 \mathrm{kpc}$. This selection cut aims to favor the selection of halo against thick-disc stars. The scale height of the thick disc has been estimated to be $\lesssim 1 \mathrm{kpc}$ at the solar radius (see figure 8 in Mateu \& Vivas 2018). The outer disc is warped and flared, and the scale height could vary by a factor of two between the Solar Neighbourhood and a distance of $12 \mathrm{kpc}$ along the plane (Amôres et al. 2017). Mateu \& Vivas (2018), by comparing with a sample of RR-Lyraes, estimated no thick-disc stars being found at $|z|>3 \mathrm{kpc}$ at the solar radius. Using the Galactic potential model GravPot16 (Fernández-Trincado et al. 2019, in prep.) that we describe in Section 2.2 below, we estimate that the number of particles at $|\mathrm{z}|>5 \mathrm{kpc}$ with respect to the total of particles at more than $4 \mathrm{kpc}$ from the Galactic Center is $2.5 \%$ for the thick disc and $47.4 \%$ for the halo.

However, Juric et al. (2008), using low-resolution SDSS data, and more recently, Wang et al (2018) from lowresolution LAMOST data, fit models showing that the stellar densities of halo and thick-disc stars at $|z| \sim 5 \mathrm{kpc}$ are still comparable. Juric et al. (2008) classified thick-disc and halo stars by their colours as a proxy for metallicity, considering halo stars those with $[\mathrm{M} / \mathrm{H}]<-1.0$. Under the light of these works it is not clear how negligible is the contribution of stars moving in thick-disc like orbits at such distances.

The above studies also show that the disc-halo transition is still not fully understood. In this framework, our target selection cut will help to better characterize the chemical, kinematical, and dynamical properties of stars at $|z|>$ $5 \mathrm{kpc}$, and help to unveil their origins.

We reject stars with flags warning of poor stellar parameter estimates, and those with signal-to-noise ratios lower than 80 . We also only consider stars with $1.0<\log g<3.5$ and $T_{\text {eff }}>4000 \mathrm{~K}$, for which the derived chemical abundances are more accurate (see Holtzmann et al. 2018, Jönsson et al.
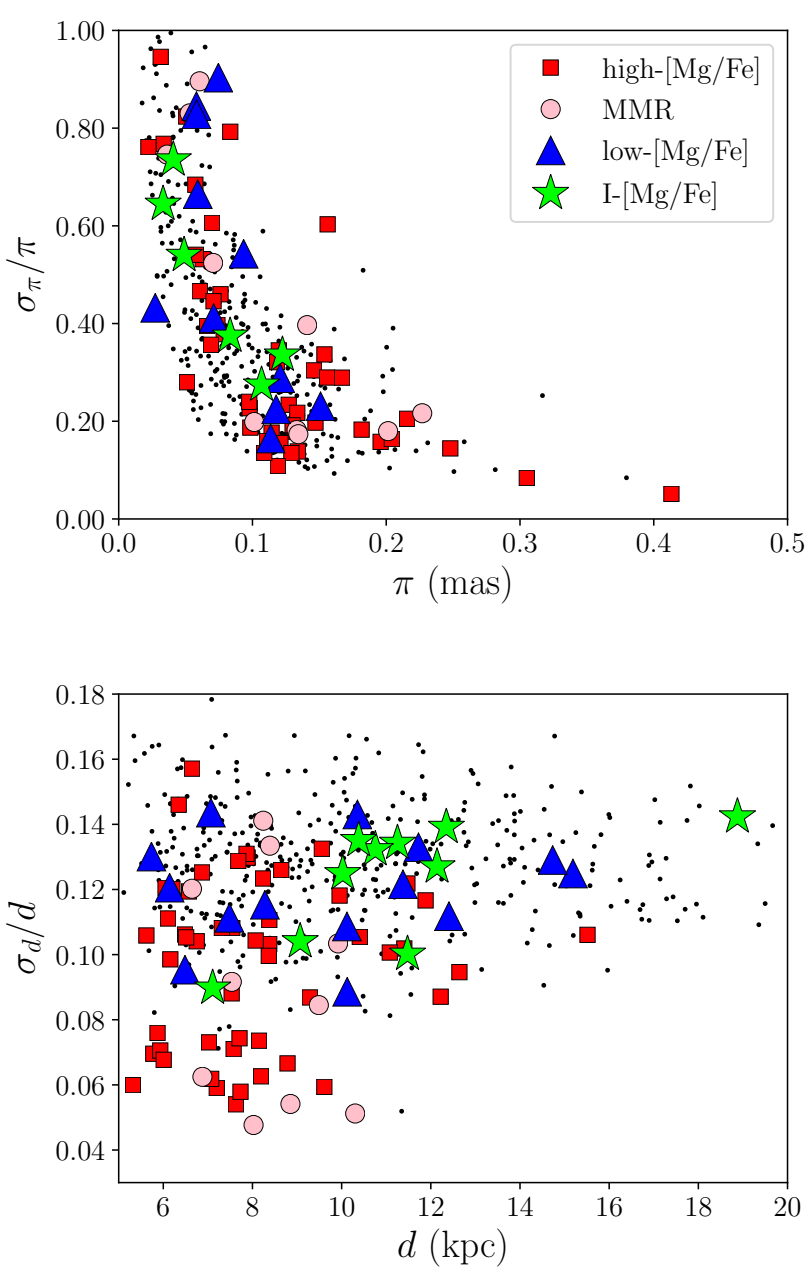

Figure 1. Top panel: Relative errors in Gaia DR2 parallaxes. Bottom panel: relative errors in the BPG distance estimates.

2018, Zasowski et al. 2018). In the following analysis we calculate orbital parameters using Gaia DR2 absolute proper motions $\left(\mu_{\alpha}\right.$ and $\left.\mu_{\delta}\right)$ and radial velocities $\left(v_{\text {rad }}\right)$ released by APOGEE DR14. The median and $\mathrm{MAD}^{1}$ of the relative errors in proper motions are $2 \pm 1 \%$ and $1 \pm 1 \%$ for $\mu_{\alpha}$ and $\mu_{\delta}$, respectively, and $0.2 \pm 0.2 \%$ for $v_{\text {rad }}$. Our final sample comprises a total of 504 stars. The coordinates, stellar parameters, $[\mathrm{Fe} / \mathrm{H}]$ and $[\mathrm{Mg} / \mathrm{Fe}]$ abundances, as well as the radial velocities, parallaxes, proper motions, and distance estimates for this sample are shown in Tables 1 and 2. This sample covers a distance to the Galactic Centre, $\left|d_{G C}\right|$, between 5 and $\sim 25 \mathrm{kpc}$. Several works have pointed out that $\sim 15-20 \mathrm{kpc}$ is a transition radius between the inner- and outer halo-regions (Carollo et al. 2007, 2010; Tissera et al. 2014; Fernández-Alvar et al. 2017). Thus, with our sample, we examine primarily the inner-halo region. 


\subsection{Distances}

Distances were calculated by the SDSS Brazilian Participation Group (BPG) using the StarHorse code described in Santiago et al. (2016) and Queiroz et al. (2018). The code calculates the posterior probability distribution over a grid of stellar evolutionary models (PARSEC 1.2S - Bressan et al. 2012) using a Bayesian methodology similar to that of Binney et al. (2014). The input parameters are the spectroscopic parameters measured by ASPCAP, Gaia DR2 parallaxes corrected for a global zero-point shift of -0.052 mas (as determined by Zinn et al. 2018), as well as APASS DR9 $B V$ gri, 2MASS $J H K_{s}$ and WISE W1W2 photometry. Thus, the distance is determined by marginalising the joint posterior PDF, not only from the parallax information, but from spectroscopic parameters and magnitudes as well. For more details, see sections 2 and 3 in Queiroz et al. (2018). The combination of Gaia DR2 and APOGEE data within the StarHorse code is described in detail in Santiago et al. (in prep.).

The priors include the space density, IMF, age distributions, and MDF of the main Galactic components (thin and thick disc, bulge, and halo). The adopted priors are as in Binney et al. (2014) (with updates in some structural parameters, from Bland-Hawthorn \& Gerhard 2016 - see Queiroz et al. 2018, section 3.3), and additional spatial priors for the bulge/bar. The age and metallicity priors are Gaussians for each Galactic component. As explained in Queiroz et al., this choice was made for simplicity, and in order to include most of the recent age and MDF found in the literature, but avoiding making the priors too especific. They assume that the impact of this choice is minor. For more details see section 3.3 in Queiroz et al. 2018.

The top panel in Figure 1 shows the fractional parallax uncertainties, and the bottom panel displays the relative errors of the distance estimates (the standard deviation divided by the mean of the probability distribution function) for our selected sample (we display stars with $[\mathrm{Fe} / \mathrm{H}]>-0.75$ with different symbols, because in the following analysis we classify stars in different groups, as we explain in Section 3.1.2). Although the relative errors in parallax are very large, the errors in distances are estimated to be less than the $20 \%$.

We quantify whether this is likely because in these cases the spectroscopic parameters and magnitudes primarily influence the distance determination. We compare them with the recent spectro-photometric distances computed by Leung \& Bovy 2019, which do not consider Gaia parallaxes as a prior. We see that, considering the whole sample, $60 \%$ of the stars show differences of less than the $30 \%$. For metalrich stars $([\mathrm{Fe} / \mathrm{H}]>-0.75$, the group that we will focuse on in this work) $80 \%$ of them show differences of less than the $20 \%$. The few stars with larger differences are not dominant in any of the different chemical groups analysed later.

We compared the BPG distances with those calculated by Bailer-Jones et al. (2018) and McMillan (2018), which also used Gaia DR2 parallaxes. In both cases, the calculated distances are from a Bayesian procedure, but the first one considers a single purely geometrical prior, while the second one also includes the radial velocity as a prior. We found a large dispersion in the comparison with the BaylerJones distances, although most of our stars show relative differences lower than the $40 \%$. There are only 81 stars with McMillan distance estimates, but the comparison is better, showing relative differences less than $20 \%$.

We repeated the analysis explained in the following sections using three different samples: $(i)$ our full stellar selection, $(i i)$ stars with relative differences with the Bayler-Jones estimates less than $40 \%$, and (iii) stars with fractional parallax uncertainties less than 50\%. Tables 4,5 , and 6 show the median and median absolute deviation obtained for velocities and orbital parameters. From inspection, the values are similar. We check that the distributions of the velocities and orbital parameters do not substantially change and, consequently, neither do the conclusions of this paper. For this reason, we maintain the analysis performed over the full sample.

\subsection{The Galactic models}

To provide a comprehensive study of our sample, we perform an orbital analysis. In order to do that, we use two non-axisymmetric models for the Galactic gravitational potential. These models are realistic (as far as possible) potentials, that fits the dynamical parameters to our best current knowledge of the Milky Way.

The first non-axisymmetric model, GravPot $16^{2}$ (Fernández-Trincado et al. 2019, in prep.), considers a 3D steady-state gravitational potential for the Galaxy, modelled as the sum of axisymmetric and non-axysimmetric components. The axisymmetric component is made-up of the superposition of seven thin discs with Einasto laws (Einasto 1979), two thick discs with sech ${ }^{2}$ laws (Robin et al. 2014), the ISM component with a density mass and a Hernquist stellar halo mass distribution like that in Robin et al. (2014). The non-axisymmetric component is a boxy/peanut bar with an assumed mass, present-day orientation and pattern speeds, which is within observational estimates that lie in the range of $1.1 \times 10^{10} \mathrm{M}_{\odot}, 20^{\circ}$, and $30-50 \mathrm{~km} \mathrm{~s}^{-1} \mathrm{kpc}$, respectively. The Sun is placed at 8.3 $\mathrm{kpc}$, the local rotation velocity is $-239 \mathrm{~km} \mathrm{~s}^{-1}$, and the Sun's orbital velocity vector $[U, V, W]_{\odot}=[-11.1,-12.24$, $7.25] \mathrm{km} \mathrm{s}^{-1}$ (Schönrich et al. 2010, Brunthaler et al. 2011). GravPot16 has been adopted in a number of recent papers (see, e.g., Fernández-Trincado et al. 2016b, 2017; Recio-Blanco et al. 2017; Albareti et al. 2017; Helmi et al. 2018, Schiappacasse-Ulloa et al. 2018; Tang et al. 2018, Contreras Ramos et al. 2018, Fernández-Trincado et al. 2019a). For a more detailed discussion, we refer the reader to a forthcoming paper (Fernández-Trincado et al. 2019, in prep.).

By combining GravPot16 with precise information from radial velocity, absolute proper motion, distance, and sky position, we ran $10^{5}$ orbit simulations for each of the metalrich halo stars, taking into account the uncertainties in the input data, where the errors were propagated as $1 \sigma$ variations in a Gaussian Monte Carlo re-sampling. For each generated set of parameters, the orbit is computed backward in time up to 2.5 Gyr. From the integrated set of orbits, we 
compute the median values for $(i) \mathrm{r}_{\text {peri }}$ and $\mathrm{r}_{\text {apo }}$, the peri/apo-galactocentric radius, respectively; (ii) the orbital eccentricity, defined as $e=\left(\mathrm{r}_{\text {apo }}-\mathrm{r}_{\text {peri }}\right) /\left(\mathrm{r}_{\text {apo }}+r_{\text {peri }}\right),($ iii $)$ the total orbital energy $E,(i v)$ the z-component of the angular momentum in the inertial frame, $L_{z}$, and $(v)$ the maximum vertical excursion from the Galactic plane, $\left|z_{\max }\right|$. The uncertainty ranges of the orbital elements are given by the $16^{\text {th }}$ and $84^{\text {th }}$ percentile value.

The second Galactic potential is based on the axisymmetric model of Allen \& Santillan (1991; AS91) using the Galactic prolate bar model (non-axisymmetric contribution) as described in Pichardo et al. (2004). The total potential has been rescaled to the Sun's Galactocentric distance and LSR velocity employed in AS91. The present orientation of the major axis of the Galactic bar is taken as $20^{\circ}$, and the angular rotation speed of the bar is $45 \mathrm{~km} \mathrm{~s}^{-1} \mathrm{kpc}^{-1}$. The results obtained with this model are consistent with GravPot16. Therefore, this second model was used to show the robustness of our analysis.

\section{ANALYSIS}

\subsection{Chemistry}

\subsubsection{The MDF as a function of $z$.}

We first examine the metallicity distribution function (MDF) as a function of the distance from the Galactic plane, $|z|$. The left panels in Figure 2 show the MDF for stars in our sample, but considering different cuts in $|z|$, i.e., those at distances $|z|>5,6, \ldots, 10 \mathrm{kpc}$. The resulting distributions exhibit peaks at $[\mathrm{Fe} / \mathrm{H}] \sim-1.4$ and extended tails at both lower and higher $[\mathrm{Fe} / \mathrm{H}]$, reaching up to solar values. In particular, there is a sample of stars at metallicities greater than $[\mathrm{Fe} / \mathrm{H}]=-0.75$, with a peak at $[\mathrm{Fe} / \mathrm{H}] \sim-0.5$, which remains even at distances $|z|>10 \mathrm{kpc}$ (although at $|z|>8$ $\mathrm{kpc}$ the high- $[\mathrm{Mg} / \mathrm{Fe}]$ population is very reduced, within the Poisson noise of the sample).

We evaluate possible selection effects due to the different cuts in color, magnitude, $T_{\text {eff }}$ and $\log g$ performed to obtain our halo sample. We reproduce these selection cuts (excluding also stars at $|z|<5 \mathrm{kpc}$ ) over a TRILEGAL simulation (Girardi et al. 2012), centered at $(l, b)=(60,75)$. This analysis reveals that the restriction to the $1.0<\log g<3.5$ range distorts the MDF, and a peak at high metallicities, larger than -1.0 , appears. For this reason we cannot rely on the shape of the MDF on the metal-rich side that we obtain from observations; however, this does not change the fact that we are detecting metal-rich stars at high distances from the plane.

The peak at $[\mathrm{Fe} / \mathrm{H}]=-1.4$ differs from the typical value associated with the MDF of the inner halo (see Carollo et al. 2007; Allende Prieto et al. 2014). We have checked if this could be due to the method employed to determine chemical abundances by the ASPCAP pipeline. The stars do not exhibit any particular trend in $T_{\text {eff }}$ and $\log g$, and homogeneously cover the stellar parameter ranges for the entire halo sample. Even if this clump of stars at $[\mathrm{Fe} / \mathrm{H}]=-1.4$ is related to some systematic effect by the abundance determinations that we are not able to detect, it does not affect our main results, since in our following analysis we focus on stars with $[\mathrm{Fe} / \mathrm{H}]>-0.75$.
The right panels of Figure 2 show the stars in the chemical space $[\mathrm{Mg} / \mathrm{Fe}]$ vs. $[\mathrm{Fe} / \mathrm{H}]$ for each sub-sample considering the different $|z|$ cuts. Those stars at $[\mathrm{Fe} / \mathrm{H}]>-0.75$ are split into different sequences of $[\mathrm{Mg} / \mathrm{Fe}]$ as a function of $[\mathrm{Fe} / \mathrm{H}]$. A significant number of stars exhibit a constant trend of larger $[\mathrm{Mg} / \mathrm{Fe}]$ values, whereas another subsample of stars follow the decreasing trend with $[\mathrm{Fe} / \mathrm{H}]$ observed for the main halo sample at lower metallicities, reaching sub-solar values. Finally, there are stars populating the space between both sequences, some of them reaching the largest $[\mathrm{Fe} / \mathrm{H}]$ values in the whole sample, $[\mathrm{Fe} / \mathrm{H}]>-0.3$.

Figure 3 shows the distribution of our sample in Galactic coordinates on an Aitoff projection. Stars in the top and bottom panels are depicted with colours indicating their $[\mathrm{Fe} / \mathrm{H}]$. Our sample, in particular the metal-rich tail, is wellspread over the sky, and exhibits no spatial evidence for being a recent accretion event. Figure 4 shows the stars in Galactic $x y$ (top panel) and $x z$ (bottom panel) coordinates, with colours indicating their $[\mathrm{Fe} / \mathrm{H}]$. Inspection of the panels shows that the metal-rich population is located at low $|z|$, extending over a wide range of $x$. As $|z|$ increases the metal-poor halo begins to dominate.

\subsubsection{The chemical characterization of the metal rich tail.}

For further analysis, we split our sample of stars at $[\mathrm{Fe} / \mathrm{H}]$ $<-0.75$ (metal-poor) and at $[\mathrm{Fe} / \mathrm{H}]>-0.75$ (metal-rich). We classify the latter considering their different $[\mathrm{Mg} / \mathrm{Fe}]$ trends with $[\mathrm{Fe} / \mathrm{H}]$. We employed the same division obtained by Hayes et al. (2018). They statistically inferred, for a sample of APOGEE DR13 metal-poor stars, $[\mathrm{M} / \mathrm{H}]$ $<-1.0$, two $[\alpha / \mathrm{Fe}]$ vs. $[\mathrm{Fe} / \mathrm{H}]$ sequences above and below a gap at $[\mathrm{Mg} / \mathrm{Fe}]=-0.2 x[\mathrm{Fe} / \mathrm{H}]$. Their sample comprises stars at lower $[\mathrm{Fe} / \mathrm{H}]$ than ours, but the work of Fernández-Alvar et al. (2018) applied this classification to a kinematically classified halo sample that covers higher metallicities, $[\mathrm{Fe} / \mathrm{H}]$ $\sim 0.0$. The Hayes et al. division cleanly separated stars into the two different $[\mathrm{Mg} / \mathrm{Fe}]$ vs. $[\mathrm{Fe} / \mathrm{H}]$ trends that the sample displayed.

Thus, we follow the same classification. However, there is a group of stars in our sample located above the division line. A later analysis of the derived orbital parameters revealed a group of intermediate $[\mathrm{Mg} / \mathrm{Fe}]$ stars that largely differs from the other stars. Consequently, we classify those stars as a different group. In addition, at $[\mathrm{Fe} / \mathrm{H}]>-0.3$ stars show lower $[\mathrm{Mg} / \mathrm{Fe}]$ values; we analyse those stars separately. Thus, the final four groups follow this classification:

(i) High- $[\mathrm{Mg} / \mathrm{Fe}]:-0.75<[\mathrm{Fe} / \mathrm{H}]<-0.3$ and $[\mathrm{Mg} / \mathrm{Fe}]>$ $+0.15$

(ii) Low- $[\mathrm{Mg} / \mathrm{Fe}]$ : $-0.75<[\mathrm{Fe} / \mathrm{H}]<-0.3$ and $[\mathrm{Mg} / \mathrm{Fe}]<$ $-0.3-0.65 *[\mathrm{Fe} / \mathrm{H}]$

(iii) Intermediate- $[\mathrm{Mg} / \mathrm{Fe}](\mathrm{I}-[\mathrm{Mg} / \mathrm{Fe}]):-0.55<[\mathrm{Fe} / \mathrm{H}]<$ -0.3 and $-0.05<[\mathrm{Mg} / \mathrm{Fe}]<+0.15$

(iv) the most metal-rich (MMR): $[\mathrm{Fe} / \mathrm{H}]>-0.3$.

In order to ensure that the differences in $[\mathrm{Mg} / \mathrm{Fe}]$ observed for the different stellar groups are physical, we investigate possible systematic errors in the abundance determinations. We compare the $[\mathrm{Mg} / \mathrm{Fe}]$ in each group with the stellar parameters $T_{\text {eff }}, \log g$ and microturbulence velocity. These parameters also play a role in the formation of the spectral lines and the under- or over-estimation of any of them can 

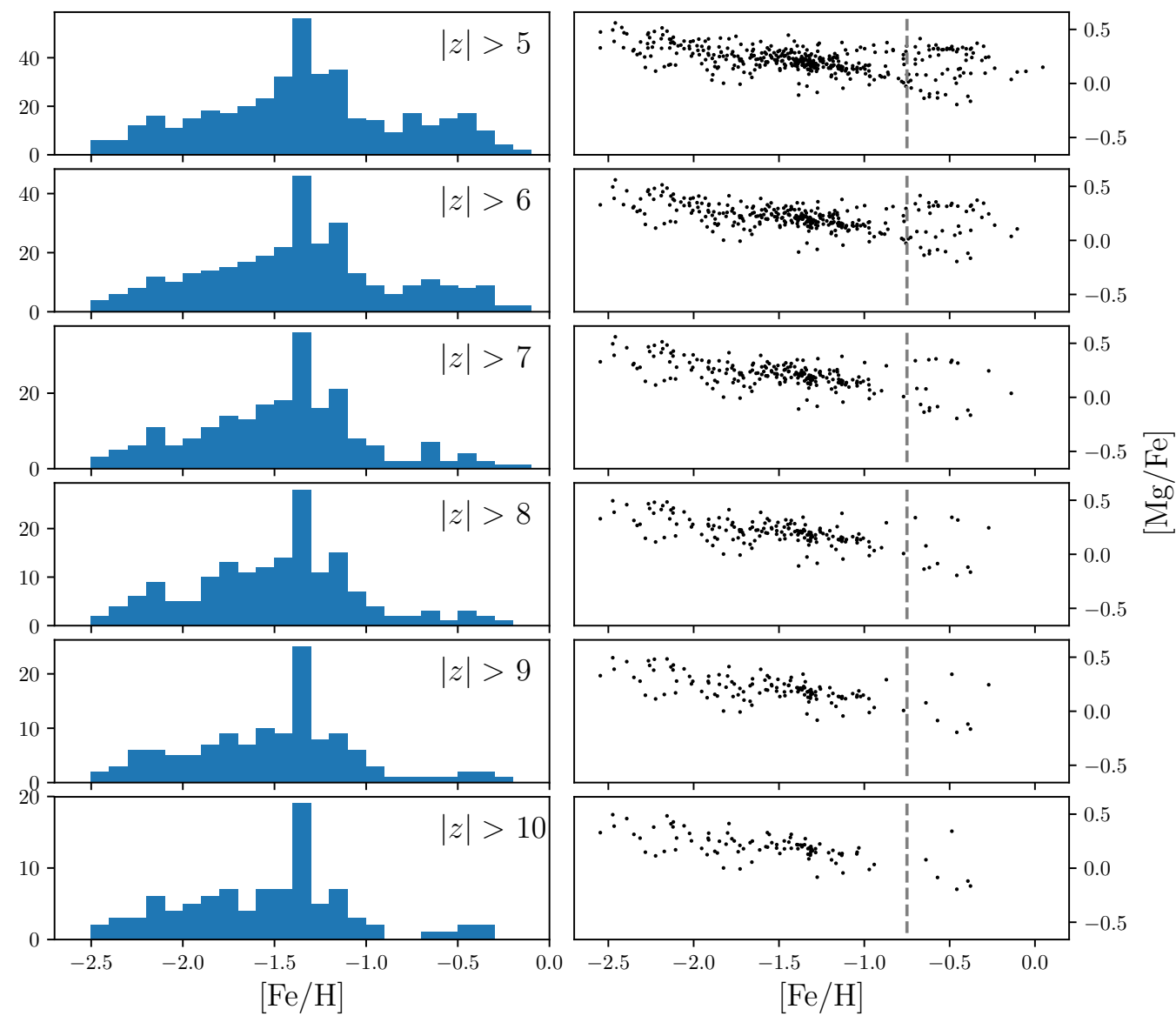

Figure 2. Left panels: Metallicity distribution functions for several subsamples of our selected APOGEE halo stars, considering different $|z|$ cuts. Right panels: $[\mathrm{Mg} / \mathrm{Fe}]$ vs. $[\mathrm{Fe} / \mathrm{H}]$ display for the corresponding subsample on the left. A vertical dashed line divides our halo sample into metal-poor and metal-rich subsamples at $[\mathrm{Fe} / \mathrm{H}]=-0.75$.

affect the derived chemical abundances. Low- $[\mathrm{Mg} / \mathrm{Fe}]$ stars show a correlation with $T_{\text {eff }}, \log g$ and an anticorrelation with $v_{\text {micro }}$, suggesting that the lower abundance values could be underestimated in cool stars. To validate the $[\mathrm{Mg} / \mathrm{Fe}]$ values, we have compared the low- $[\mathrm{Mg} / \mathrm{Fe}]$ spectra with those of $\mathrm{I}-[\mathrm{Mg} / \mathrm{Fe}]$ and high- $[\mathrm{Mg} / \mathrm{Fe}]$ twins (i.e., diferences in their measured $T_{\text {eff }}, \log g,[\mathrm{Fe} / \mathrm{H}]$ and $v_{\text {micro }}$ lower than $80 \mathrm{~K}, 0.34$ dex, 0.13 dex and $0.2 \mathrm{kms}^{-1}$, respectively). This comparison shows that the $\mathrm{Mg}$ lines used to derive the abundances are in fact weaker for the low- $[\mathrm{Mg} / \mathrm{Fe}]$ stars, confirming their lower abundance. No other correlations were found between the $[\mathrm{Mg} / \mathrm{Fe}]$ with any other parameter, indicating that the chemical differences of the stellar groups are not due to systematic effects.

We also explore other chemical species. The panels in Figure 6 show the chemical trends of $\alpha$-element-to-iron ratios: $[\mathrm{O} / \mathrm{Fe}],[\mathrm{Mg} / \mathrm{Fe}],[\mathrm{Si} / \mathrm{Fe}],[\mathrm{S} / \mathrm{Fe}],[\mathrm{Ca} / \mathrm{Fe}]$, and $[\mathrm{Ti} / \mathrm{Fe}]$ as a function of $[\mathrm{Fe} / \mathrm{H}]$. The largest differences in the chemical trends are observed in $[\mathrm{Mg} / \mathrm{Fe}]$, but for the other $\alpha$-elements the high- $[\mathrm{Mg} / \mathrm{Fe}]$ (and low- $[\mathrm{Mg} / \mathrm{Fe}]$ ) stars also show higher (and lower) values than the $\mathrm{I}-[\mathrm{Mg} / \mathrm{Fe}]$ and MMR stars. As observed by Nissen \& Schuster (2010), the differences are milder for Ca than for the other $\alpha$-elements.

It is worth to emphasize that ASPCAP pipeline provides chemical abundance ratios under the assumption of local thermodynamic equilibrium (LTE), and therefore possible non-LTE (NLTE) and/or 3D effects on the derived values of the atmospheric parameters and chemical abundances are not taken into account. There are hints of these effects in some APOGEE stars (see Hawkins et al. 2016, for instance).

Bergemann et al. 2017b estimated that $<3$ D $>$ NLTEcorrections in metal-poor giants should be of the order of $\sim 0.1-0.2 \mathrm{dex}$, in the $T_{\text {eff }}$ regime of our objects from measurements in the optical range. However, they found that their resulting $[\mathrm{Mg} / \mathrm{Fe}]$ distribution with $[\mathrm{Fe} / \mathrm{H}]$ taking into account $<3 \mathrm{D}>$ NLTE effects is consistent with the one obtained by ASPCAP from estimates in the infrarred H-band considering LTE, mainly at high metallicities (see Fig. 5d and Fig. 6 in Bergemann et al.). This encourages thinking that these effects in ASPCAP measurements for our stars should be small. In addition, there is a recent study by Zhang et al. (2017) which evaluated the NLTE effects in 


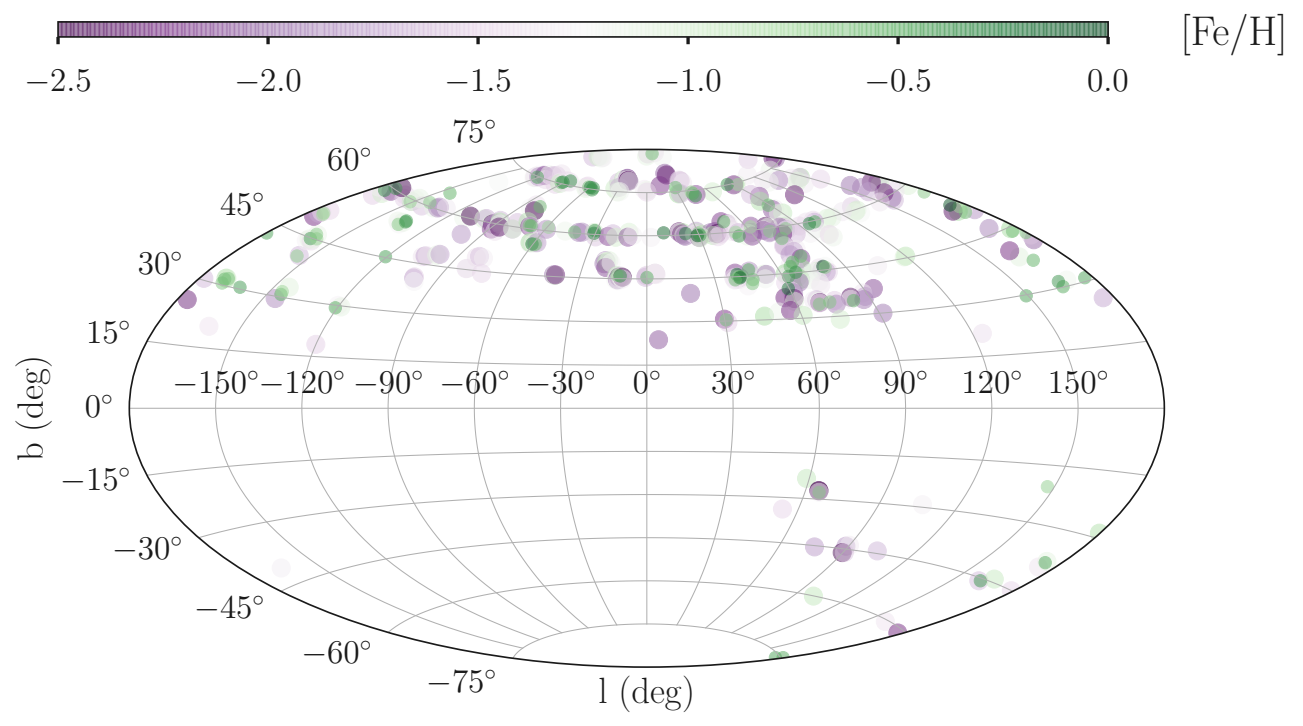

Figure 3. Distribution of our halo sample in Galactic coordinates on an Aitoff projection. The stars are colour-coded by [Fe/H].

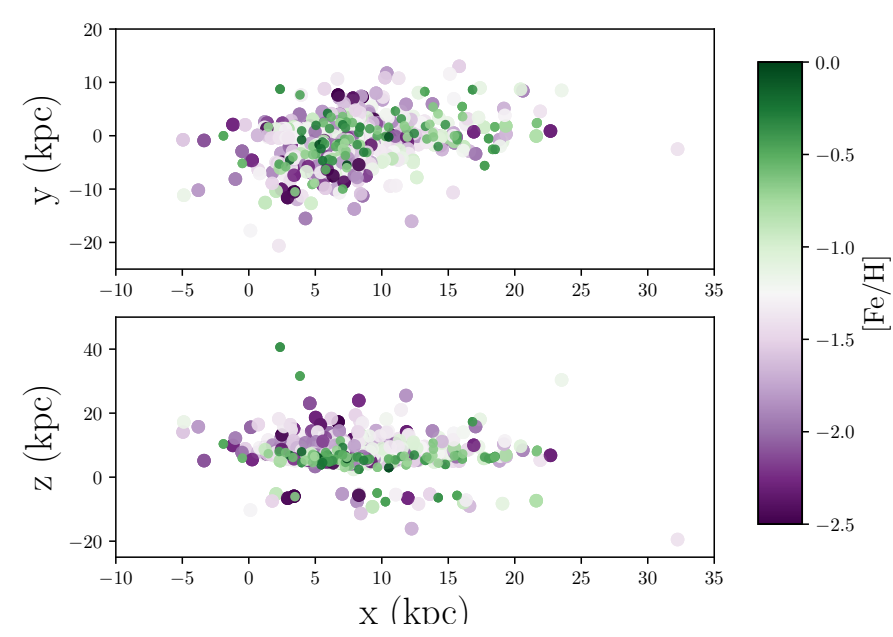

Figure 4. Distribution of our halo sample in Galactic $x y$ and $x z$ coordinates. $(x, y, z)$ form a right-handed system, with $x$ positive towards the Sun, $z$ towards the North Galactic Pole, and $y$ in the direction opposite to Galactic rotation. The colour scale indicates the $[\mathrm{Fe} / \mathrm{H}]$ of each star, as in Figure 3.

$\mathrm{Mg}$ lines in the H-band. The resulting $[\mathrm{Mg} / \mathrm{Fe}]$ abundance differences considering LTE and NLTE for the thirteen stars that they compared were lower than 0.1 except for one star, and in the worse case the correction reaches up to $\sim-0.22$. The $[\mathrm{Mg} / \mathrm{Fe}]$ values displayed by our high- $[\mathrm{Mg} / \mathrm{Fe}]$ and low$[\mathrm{Mg} / \mathrm{Fe}]$ exceed this value, suggesting that the bimodality is not explained only by these effects. We are aware of that a $<3$ D $>$ NLTE analysis is important to well-establish the chemical differences between stellar populations. But this kind of analysis is beyond the scope of this paper.

Figure 6 displays the trends with $[\mathrm{Fe} / \mathrm{H}]$ for other elements derived by ASPCAP, with a different nucleosynthetic origin: $[\mathrm{Ni} / \mathrm{Fe}],[\mathrm{K} / \mathrm{Fe}],[\mathrm{Al} / \mathrm{Fe}],[\mathrm{Na} / \mathrm{Fe}],[\mathrm{Mn} / \mathrm{Fe}]$, and
$[\mathrm{Cr} / \mathrm{Fe}]$. As in previous works, the dichotomy between high$[\mathrm{Mg} / \mathrm{Fe}]$ and low- $[\mathrm{Mg} / \mathrm{Fe}]$ stars is also observed in our data for $[\mathrm{Ni} / \mathrm{Fe}]$, and also $[\mathrm{Al} / \mathrm{Fe}]$ and $[\mathrm{K} / \mathrm{Fe}]$ (see Nissen \& Schuster 2010; Fernández-Alvar et al. 2017). Nissen \& Schuster detected the same dichotomy in $[\mathrm{Na} / \mathrm{Fe}]$, however the ASP$\mathrm{CAP}[\mathrm{Na} / \mathrm{Fe}]$ estimates exhibit a very large dispersion and no clear trend with $[\mathrm{Fe} / \mathrm{H}]$. These estimates can be affected by strong telluric absorption near the abundance windows in the stars (Zasowski et al. 2018). Thus, they should be considered with caution. Nissen \& Schuster (2011) found that their high- and low- $\alpha$ populations were indistinguishable in $[\mathrm{Mn} / \mathrm{Fe}]$ and $[\mathrm{Cr} / \mathrm{Fe}]$ trends with $[\mathrm{Fe} / \mathrm{H}]$. Our results show that all the populations overlap, although the low- $[\mathrm{Mg} / \mathrm{Fe}]$ population tends to have lower $[\mathrm{Mn} / \mathrm{Fe}]$ and $[\mathrm{Cr} / \mathrm{Fe}]$ values.

The I- $[\mathrm{Mg} / \mathrm{Fe}]$ and MMR groups show values in between the high- and low- $[\mathrm{Mg} / \mathrm{Fe}]$ stars for all the $\alpha$-elements (except $[\mathrm{S} / \mathrm{Fe}])$, and $[\mathrm{Ni} / \mathrm{Fe}],[\mathrm{K} / \mathrm{Fe}]$, and $[\mathrm{Al} / \mathrm{Fe}]$. In the case of $[\mathrm{Mn} / \mathrm{Fe}]$ and $[\mathrm{Cr} / \mathrm{Fe}]$, the $\mathrm{I}-[\mathrm{Mg} / \mathrm{Fe}]$ overlap with the high$[\mathrm{Mg} / \mathrm{Fe}]$ group, and the MMR stars follow the same trend as the high- $[\mathrm{Mg} / \mathrm{Fe}]$ stars with $[\mathrm{Fe} / \mathrm{H}]$.

The differences observed in the abundance of several elements, some of which previous studies also detected a dichotomy in their abundances, underscore the conclusion that the stars that we are analysing at high metallicities are chemically diverse.

\subsubsection{Belonging to known streams?}

In order to test if some of the metal-rich halo we identify belongs to a known stream, we compare the spatial distribution of our halo sample with the distances and positions of all the streams currently detected in the halo. Some of them match the path of the Sagittarius stream in Galactic coordinates, and some stars in our sample are located at distances compatible to its trailing arm. At $[\mathrm{Fe} / \mathrm{H}]>-0.75$, Sagittarius stars exhibit similar $[\mathrm{Mg} / \mathrm{Fe}]$ values (Hasselquist et al. 2017 ) as most of our low- $[\mathrm{Mg} / \mathrm{Fe}]$ metal-rich halo stars. We also compared the abundance ratios $[\mathrm{O} / \mathrm{Fe}],[\mathrm{Si} / \mathrm{Fe}],[\mathrm{Ca} / \mathrm{Fe}]$, 

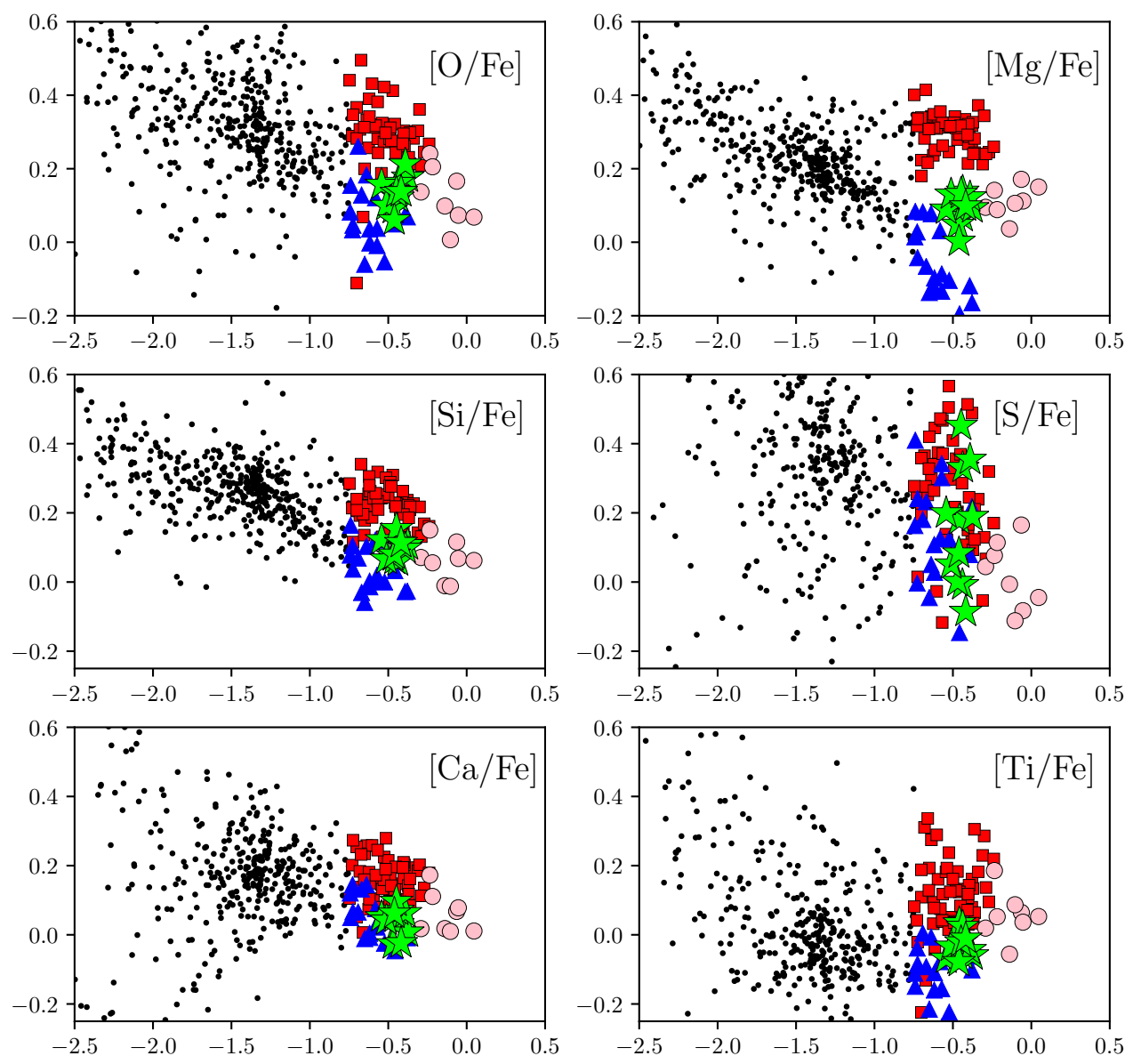

$[\mathrm{Fe} / \mathrm{H}]$

Figure 5. $[\mathrm{X} / \mathrm{Fe}]$, as a function of $[\mathrm{Fe} / \mathrm{H}]$, for the $\alpha$-elements $\mathrm{O}, \mathrm{Mg}, \mathrm{Si}, \mathrm{S}, \mathrm{Ca}$, and Ti. The metal-poor sample is plot with black dots, whereas the metal-rich stellar groups are depicted with the same symbols as in Figure 1.

$[\mathrm{Ni} / \mathrm{Fe}],[\mathrm{Al} / \mathrm{Fe}],[\mathrm{K} / \mathrm{Fe}]$, and $[\mathrm{Mn} / \mathrm{Fe}]$, and find that a number of stars (those with $[\mathrm{Mg} / \mathrm{Fe}]<0.0$ ) in our low- $[\mathrm{Mg} / \mathrm{Fe}]$ halo sample agree in those abundance ratios with those measured for Sagittarius stars at the same metallicities.

Comparison of the GRV ${ }^{3}$ (in the range $[-100,100]$ $\mathrm{kms}^{-1}$ ) with Carlin et al. (2018) shows an agreement with their GRV measurements for Sgr. For these reasons, we conclude that stars with $[\mathrm{Mg} / \mathrm{Fe}]<0.0$ in our low- $[\mathrm{Mg} / \mathrm{Fe}]$ population are likely associated with the Sagittarius stream.

We notice that the $\mathrm{I}-[\mathrm{Mg} / \mathrm{Fe}]$ group matches in $(l, b)$, as well as in chemistry, with the two stellar overdensities in Triangulum/Andromeda (Rocha-Pinto et al. 2004; Martin et al. 2007; Price-Whelan et al. 2015; Perottoni et al. 2018) and A13 (Sharma et al. 2010; Li et al. 2017). These have been recently spectrocopically followed up by Bergemann et al. (2018) and Hayes et al. (2018b). The latter an-

3 The radial velocity $v_{\text {rad }}$ corrected for solar motion (we adopt the solar Galactocentric velocities $U_{\odot}=11.1 \mathrm{kms}^{-1}, V_{\odot}=12.24 \mathrm{kms}^{-1}$ and $W_{\odot}=7.25 \mathrm{kms}^{-1}$, as in Schönrich et al. 2010). alyzed APOGEE stars specifically targeted to explore the Triaungulum/Andromeda overdensity. However, none of our $\mathrm{I}-[\mathrm{Mg} / \mathrm{Fe}]$ stars are one of these targets. In the following kinematical and dynamical analysis we will examine whether our I- $[\mathrm{Mg} / \mathrm{Fe}]$ stars belong to the same stellar population as these two overdensities, and discuss the implications of our results for this explanation of their origin.

In summary, our sample of halo candidates observed by APOGEE with $|z|>5 \mathrm{kpc}$ and $5<\left|d_{G C}\right|<25 \mathrm{kpc}$ ranges in metallicity over $-2.5<[\mathrm{Fe} / \mathrm{H}]<0.0$. These stars exhibit a main decreasing trend of $[\mathrm{Mg} / \mathrm{Fe}]$ (and other $\alpha$-elements) with $[\mathrm{Fe} / \mathrm{H}]$, from the most metal-poor stars up to $[\mathrm{Fe} / \mathrm{H}]$ $\sim-1.0$, where a split in several trends is evident. In other words, the metallicitiy distribution function of the stars populating the region at more than $5 \mathrm{kpc}$ from the Galactic plane shows a metal-rich tail, comprised of several chemically distinct stellar populations, pointing to different origins.

A dichotomy in the $[\alpha / \mathrm{Fe}]$ vs. $[\mathrm{Fe} / \mathrm{H}]$ space had already been detected in several previous works (Nissen \& Schuster 2010; Recio-Blanco et al. 2014 ; Fernández-Alvar et al. 2018; 

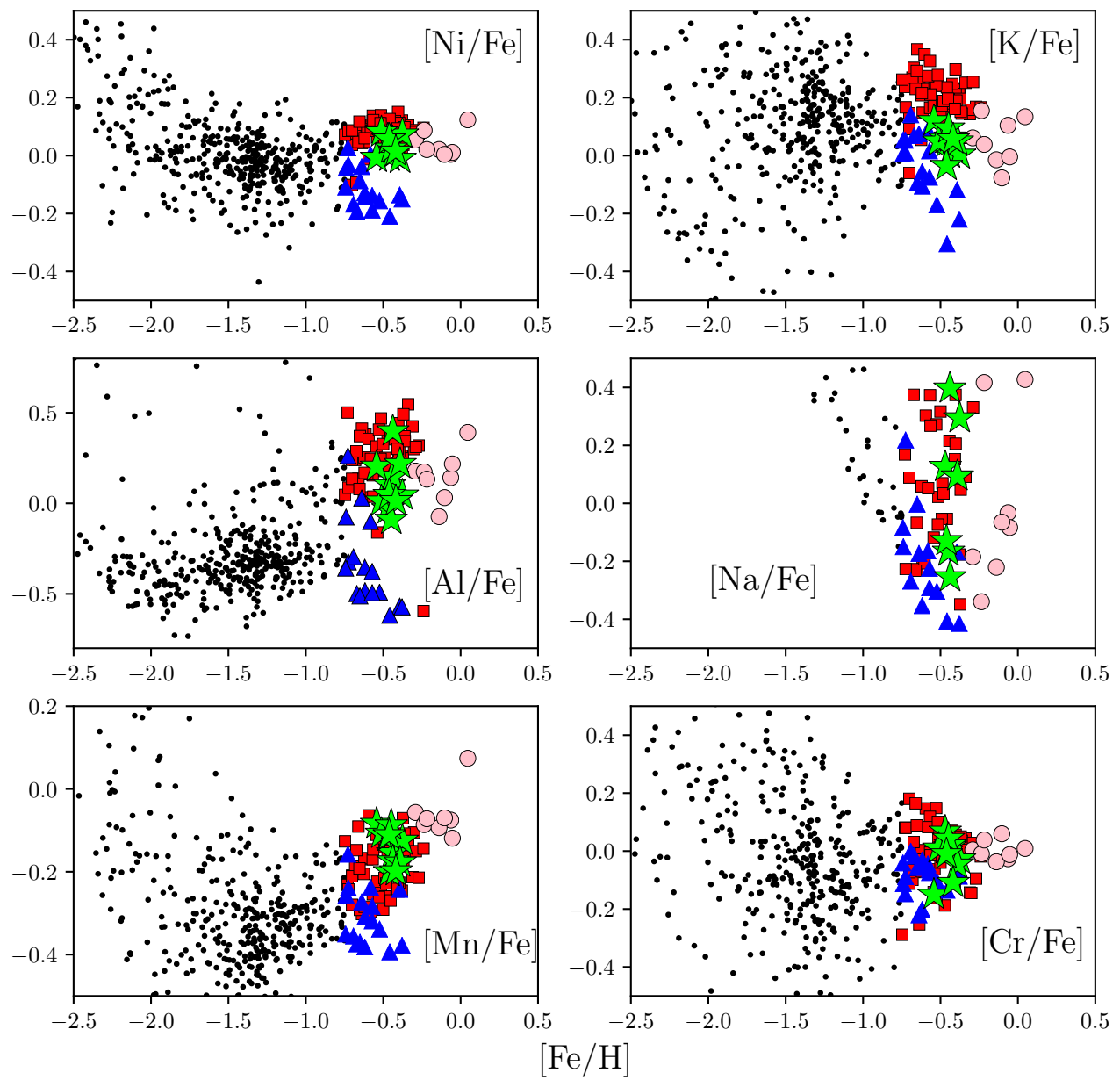

Figure 6. $[\mathrm{X} / \mathrm{Fe}]$ as a function of $[\mathrm{Fe} / \mathrm{H}]$ for $\mathrm{Ni}, \mathrm{K}, \mathrm{Al}, \mathrm{Na}, \mathrm{Mn}$ and $\mathrm{Cr}$. The metal-poor sample is plot with black dots, whereas the metal-rich stellar groups are depicted with the same symbols as in Figure 1.

Hayes et al. 2018a). However, this is the first time the distribution of this metal-rich tail with $|z|$ is explored. Our sample reveals that the split into chemically distinct stellar populations reaches distances up to $|z|>5 \mathrm{kpc}$ from the Galactic plane.

\subsection{Kinematics}

Figure 7 displays the galactocentric velocity components, $V_{x}$, $V_{y}$ and $V_{z}$, derived for our sample in a Toomre diagram. The metal-poor stars are plotted as black points, whereas the metal-rich stellar groups are depicted using different symbols as defined in Section 3.1, and have colours corresponding to their $[\mathrm{Fe} / \mathrm{H}]$ values. The orange and green lines indicate constant space velocities of $70 \mathrm{kms}^{-1}$ and $180 \mathrm{kms}^{-1}$, respectively, which illustrate the boundaries within which the thick-disc stars more likely dominate (Fuhrmann 1999; Soubiran et al. 2003). The exact values are not deterministic. It is only the relative ordering that contains physical information (i.e. larger asymmetric drift and velocity dispersion for the thick-disc relative to the thin-disc). Thus, they are only a reference. But we would like to consider them because these boundaries have been used in several works which aimed to characterize the thin- and thick-discs and the halo (Venn et al. 2004, Nissen 2004, Nissen \& Schuster 2010, Bensby et al. 2014, Anders et al. 2014). The red line shows a constant Galactic rest-frame velocity of $533 \mathrm{kms}^{-1}$.

The entire low- $[\mathrm{Mg} / \mathrm{Fe}]$ population (except for one star) and most of the high- $[\mathrm{Mg} / \mathrm{Fe}]$ and MMR stars are located outside the thick-disc boundary, i.e., they show halo-like total space velocities, although some of the high- $[\mathrm{Mg} / \mathrm{Fe}]$ and MMR stars have lower velocities, compatible with disc kinematics. Noticeably, the I- $[\mathrm{Mg} / \mathrm{Fe}]$ stars are more concentrated in the diagram, in contrast with the large dispersions displayed by the other stellar groups, and overlay the velocity values delimiting the thin/thick-disc components.

Consequently, not all our halo candidates show halolike kinematics. However, except for the I- $[\mathrm{Mg} / \mathrm{Fe}]$ group, there are high- $[\mathrm{Mg} / \mathrm{Fe}], \mathrm{MMR}$ and low- $[\mathrm{Mg} / \mathrm{Fe}]$ stars moving with halo-like velocities, i.e., $V_{\text {tot }}>200 \mathrm{~km} / \mathrm{s}$. We check that considering only these more likely halo stars based on their 


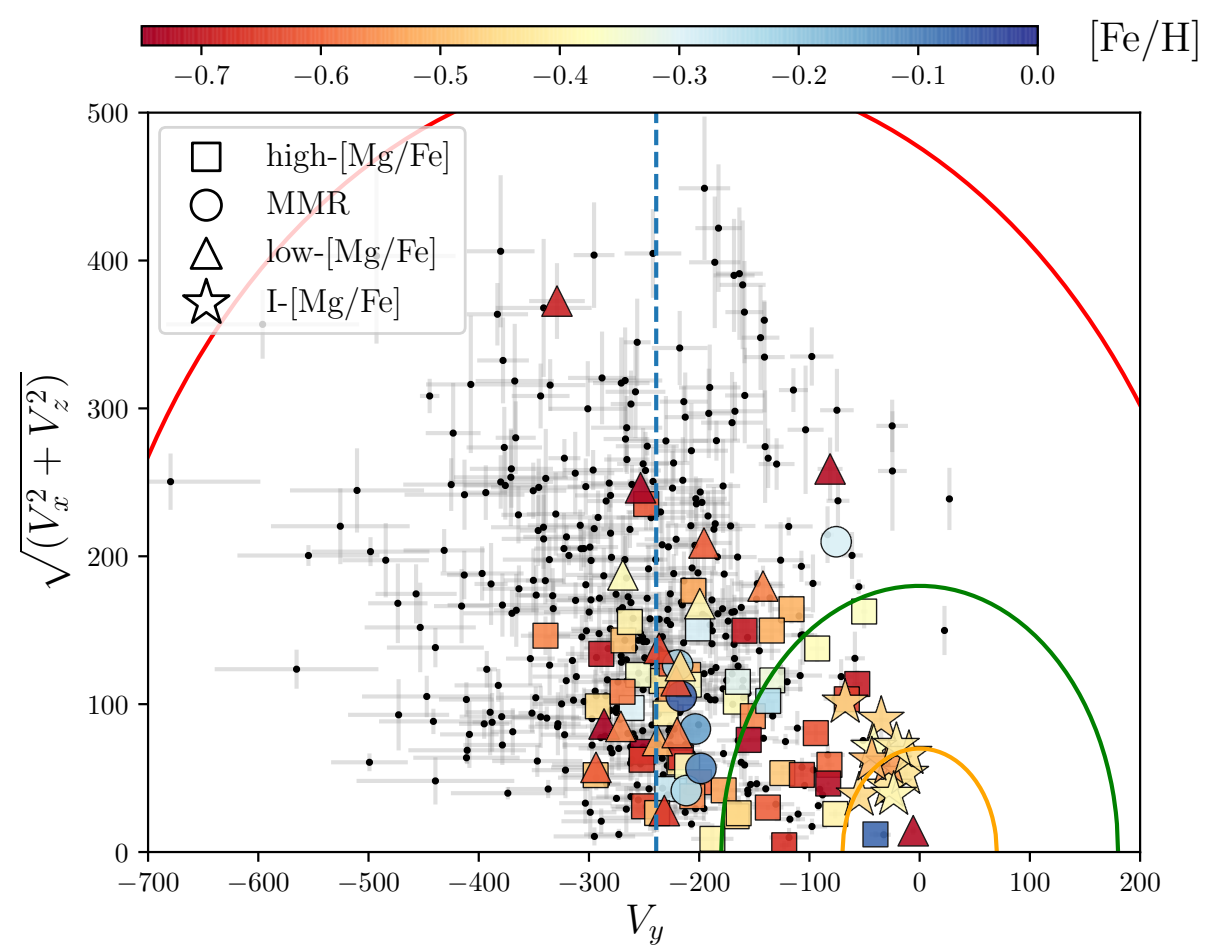

Figure 7. Toomre diagram for the metal-poor halo sample (black dots with error bars) and metal-rich halo sample (with colours indicating the metallicity, $[\mathrm{Fe} / \mathrm{H}])$. The metal-rich groups are highlighted with different symbols: squares (high- $[\mathrm{Mg} / \mathrm{Fe}]$ ), circles (MMR), triangles (low- $[\mathrm{Mg} / \mathrm{Fe}]$ ) and stars $(\mathrm{I}-[\mathrm{Mg} / \mathrm{Fe}])$. Red line: Constant Galactic rest-frame velocity of $533 \mathrm{~km} \mathrm{~s}^{-1}$; green line: Thick-disc constant velocity of $180 \mathrm{~km} \mathrm{~s}^{-1}$ (Soubiran et al. 2003); orange line: Thin-disc constant velocity of $70 \mathrm{~km} \mathrm{~s}^{-1}$ (Fuhrmann 1999). The vertical blue dashed line indicates zero Galactic rotation, $\mathrm{V}_{l s r}=-239 \mathrm{~km} \mathrm{~s}^{-1}$.

kinematics we still see the $[\mathrm{Mg} / \mathrm{Fe}]$ vs. $[\mathrm{Fe} / \mathrm{H}]$ dichotomy up to distances $>8 \mathrm{kpc}$.

We now examine in more statistical detail how the different metal-rich groups are distributed in the Toomre diagram. We performed a Kolmogorov-Smirnov test to compare the $V_{y}$ velocity component distributions for the five groups defined above. The test carried out over the metalrich groups results in the strong rejection of the $\mathrm{I}-[\mathrm{Mg} / \mathrm{Fe}]$ stars belonging to the same distribution as any of the other groups ( $p$-value $<0.001)$.

Comparing with the metal-poor sample, the statistical tests shows that the $\mathrm{I}-[\mathrm{Mg} / \mathrm{Fe}]$ stars do not belong to the same distribution as the metal-poor sample ( $p$-value $<$ $0.001)$. Although slightly less significantly, the high- $[\mathrm{Mg} / \mathrm{Fe}]$ stars $(p$-value $=0.02)$, the most metal-rich stars $(p$-value $=$ $0.01)$, and the low- $[\mathrm{Mg} / \mathrm{Fe}]$ group $(\mathrm{p}$-value $=0.08)$ also exhibit $V_{y}$ velocity distributions that differ from that of the metal-poor group, suggesting the latter is dominated by stars with a different kinematical behaviour than the metalrich group.

The panels in Figure 8 are histograms of the distribution of high- $[\mathrm{Mg} / \mathrm{Fe}]$ (comparing with the MMR group of stars) and low- $[\mathrm{Mg} / \mathrm{Fe}]$ (comparing with $\mathrm{I}-[\mathrm{Mg} / \mathrm{Fe}]$ stars) for $V_{y}$ and $\sqrt{V_{x}^{2}+V_{z}^{2}}$. Overall, this analysis reveals that the high$[\mathrm{Mg} / \mathrm{Fe}]$ group has a large fraction of stars with prograde orbits, but also a tail with retrograde orbits. The low- $[\mathrm{Mg} / \mathrm{Fe}]$ stars are more skewed, and distributed around $V_{y} \sim-239$ $\mathrm{kms}^{-1}$. The I-[Mg/Fe] stars stand out as a group moving with a $V_{y}$ similar to the rotation of the Galactic disc.
The distribution of $\sqrt{V_{x}^{2}+V_{z}^{2}}$ is also different between both groups - high- $[\mathrm{Mg} / \mathrm{Fe}]$ stars are characterized by a distribution with two peaks at $\sim 50 \mathrm{kms}^{-1}$ and $\sim 125$ $\mathrm{kms}^{-1}$. The low-[Mg/Fe] group exhibits a broader distribution, reaching larger values, up to $\sim 300 \mathrm{kms}^{-1}$. The I$[\mathrm{Mg} / \mathrm{Fe}]$ group is characterized by low $\sqrt{V_{x}^{2}+V_{z}^{2}}$. The median and MAD values of $V$ and $\sqrt{V_{x}^{2}+V_{z}^{2}}$ for each group are shown in Table 4

These results highlight that the chemically distinct stellar groups are also characterized by a different kinematical signature. It is important to notice, however, that several previous works have pointed out that a kinematical classification of stars in the different Galactic components using the Toomre diagram do not cleanly separate them (see Schönrich \& Binney 2009, for instance). Different chemically identified components exhibit an overlap of their velocity distributions. In addition, comparison of the stellar velocity components $\left(V_{x}, V_{y}, V_{z}\right)$ at present time may not always be a good indicator of the kind of orbit described by the star, and thus the Galactic component to which it belongs, since this orbit changes with time. Orbital parameters integrated over time are a more reliable aproach, as we describe in the next section. 

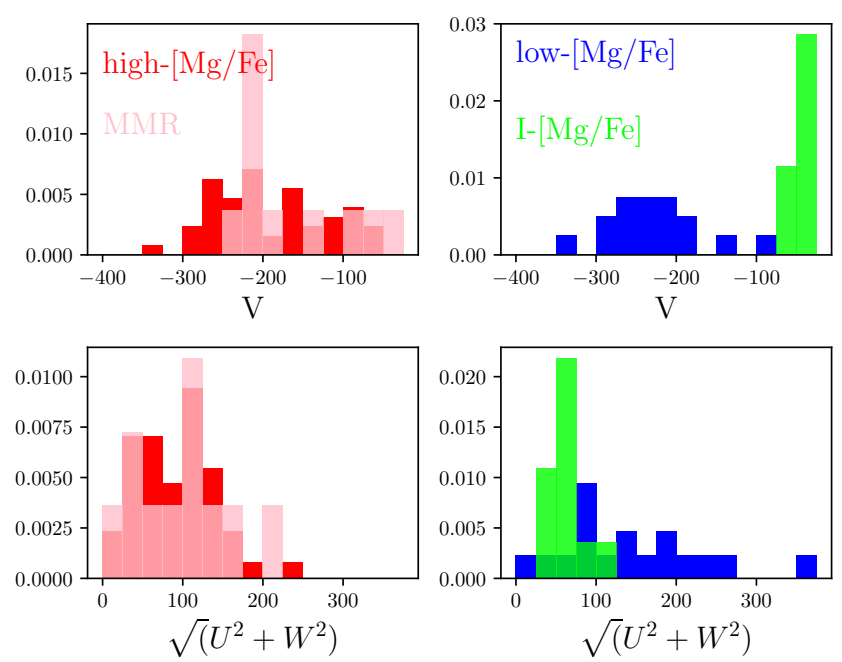

Figure 8. Top panels: Distribution of the $V_{y}$ velocity component for stars with $-0.75<[\mathrm{Fe} / \mathrm{H}]<-0.3$ and $[\mathrm{Mg} / \mathrm{Fe}]>+0.2$ (high$[\mathrm{Mg} / \mathrm{Fe}]+\mathrm{MMR}-$ left panel $)$, and for stars with $-0.75<[\mathrm{Fe} / \mathrm{H}]$ $<-0.3$ and $[\mathrm{Mg} / \mathrm{Fe}]<+0.2$ (low- $[\mathrm{Mg} / \mathrm{Fe}]+\mathrm{I}-[\mathrm{Mg} / \mathrm{Fe}]-$ right panel). Bottom panels: Distribution of the $\sqrt{V_{x}^{2}+V_{z}^{2}}$ for the same subsamples of stars as in the upper panels.

\subsection{Dynamical properties as a function of chemistry}

Tables 5 and 6 shows the median values of the orbital elements derived for each group, revealing that our chemically distinct groups show also a different dynamical behaviour. To better visualize this, we plot in Figure 9 our sample in action space ( $E$ vs. $L_{z}$ ) color coded by $[\mathrm{Mg} / \mathrm{Fe}]$ and with the same symbols used in previous Figures for each metal-rich stellar group, and black dots showing the metal-poor stars. Figures 10 and 11 display the apocenters, pericenters and maximum z $\left(r_{\text {apo }}, r_{\text {peri }}\right.$ and $\left.\left|z_{\max }\right|\right)$ reached during the orbits as a function of $[\mathrm{Fe} / \mathrm{H}]$, accompanied by histograms. Finally, Figure 12 shows the trends of the total orbital energy, $E$, and the z-component of the angular momentum, $L_{z}$ with $\left|z_{\max }\right|$, with colours indicating the orbital eccentricity, $e$.

The high- $[\mathrm{Mg} / \mathrm{Fe}]$ stars are characterized by lower energies, i.e., they are more bound to the Galaxy than the low- $[\mathrm{Mg} / \mathrm{Fe}]$ stars, and a more prograde median angular momentum, although some stars show no rotation or even a retrograde motion. In addition, the orbits of the high- $[\mathrm{Mg} / \mathrm{Fe}]$ stars tend to remain at lower $\left|z_{\max }\right|$ than the low- $[\mathrm{Mg} / \mathrm{Fe}]$ stars.

The low- $[\mathrm{Mg} / \mathrm{Fe}]$ population exhibits a broader range of energies, apocenters and maximum distances from the plane, reaching higher values than the other groups, and are more symmetrically distributed around $L_{z}=0$. Both the high- $[\mathrm{Mg} / \mathrm{Fe}]$ and low- $[\mathrm{Mg} / \mathrm{Fe}]$ populations are characterized by large eccentricities, $e \sim 0.8$ (except a few high- $[\mathrm{Mg} / \mathrm{Fe}]$ stars which follow the locus of the disc in action space, and consequently extend toward lower eccentricities). Compared to these metal-rich stars, there are more stars within the metal-poor group moving on retrograde orbits, with lower eccentricities and higher energies.

There are trends of the total orbital energy and the z- component of the angular momentum with maximum $\left|z_{\max }\right|$ reached during the orbits, for both stellar populations. In the case of the high- $[\mathrm{Mg} / \mathrm{Fe}]$ population, stars that reach higher $\left|z_{\max }\right|$ during their orbits are less bound, have an angular momentum closer to 0 . The low- $[\mathrm{Mg} / \mathrm{Fe}]$ stars exhibit the same trends for $E$, but the opposite behaviour for $L_{z}$ with $\left|z_{\max }\right|$.

It is evident that the orbits for the $\mathrm{I}-[\mathrm{Mg} / \mathrm{Fe}]$ group differ greatly from the other groups. They are characterized by prograde orbits with low eccenticities, reaching relatively low $\left|z_{\max }\right|$ but with large energies (i.e., they are less bound). These stars are those with the most prograde orbits within all our stars at high $|z|$. Noticeable is that the I- $[\mathrm{Mg} / \mathrm{Fe}]$ stars reach the largest $r_{p e r i}$, in concordance with a possible origin in the outer parts of the disc.

A Kolmogorov-Smirnov test shows that, comparing the four metal-rich groups in $E$ and $\left|z_{\max }\right|$, the hypothesis of belonging to the same distribution can be rejected in the case of the high- $[\mathrm{Mg} / \mathrm{Fe}]$ stars compared with the low- $[\mathrm{Mg} / \mathrm{Fe}]$ stars, but not compared with the MMR stars, supporting the hypothesis that the MMR stars belong to the same stellar population as the high- $[\mathrm{Mg} / \mathrm{Fe}]$ stars. This statistical test also reveals that the $\mathrm{I}-[\mathrm{Mg} / \mathrm{Fe}]$ stars have a very different distribution (with $p$-values $<0.001$ ) in every orbital parameter compared with both the high- $[\mathrm{Mg} / \mathrm{Fe}]$ and low- $[\mathrm{Mg} / \mathrm{Fe}]$ populations.

In summary, our analysis reveals that the chemically distinct groups describe orbits that differ from one group to the other. Examples of stellar orbits computed for one high$[\mathrm{Mg} / \mathrm{Fe}]$, one I- $[\mathrm{Mg} / \mathrm{Fe}]$ stars and one low- $[\mathrm{Mg} / \mathrm{Fe}]$ stars, in the $|z|$ vs. $\mathrm{R}$ (radius projected onto the Galactic plane) and $y$ vs. $x$ planes, are shown in Figure 1 in the Appendix Section.

\section{THE ORIGIN OF OUR HIGH-|Z METAL-RICH POPULATIONS.}

The metal-rich tail observed in our halo candidates sample splits into three different $[\mathrm{Mg} / \mathrm{Fe}]$ trends with $[\mathrm{Fe} / \mathrm{H}]$. This is not the first time that different sequences in the $[\alpha / \mathrm{Fe}]$ vs. $[\mathrm{Fe} / \mathrm{H}]$ were detected. Nissen \& Schuster $(2010,2011)$ identified two distinct stellar populations in a local volume, within 350 pc centreed on the Sun. Hayes et al. (2018a) statistically verified that these two chemically distinct trends were the dominant populations for stars with $[\mathrm{Fe} / \mathrm{H}]<-0.9$. Fernández-Alvar et al. (2018) revealed the same two distinct trends, selecting halo stars by their kinematics (large radial velocities), and covering Galactocentric radii up to beyond $20 \mathrm{kpc}$. This is also the case in the recent Haywood et al. (2018) work. However, this is the first time we detect a high-/low- $\alpha$ dichotomy in metal-rich stars, $[\mathrm{Fe} / \mathrm{H}]>-0.75$, located at distances $>5 \mathrm{kpc}$ from the Galactic plane. Are we looking at the same stellar populations detected before?

\subsection{The low- $[\mathrm{Mg} / \mathrm{Fe}]$ and the metal-poor stars.}

Nissen $\&$ Schuster (2010) found that their high- $\alpha$ stars tend to have prograde orbits, whereas low- $\alpha$ stars show preferentially retrograde orbits. Schuster et al. (2012) also found differences between the high- $\alpha$ and low- $\alpha$ orbital parameters - the former showed typical values of $r_{a p o} \sim 16 \mathrm{kpc}$, 


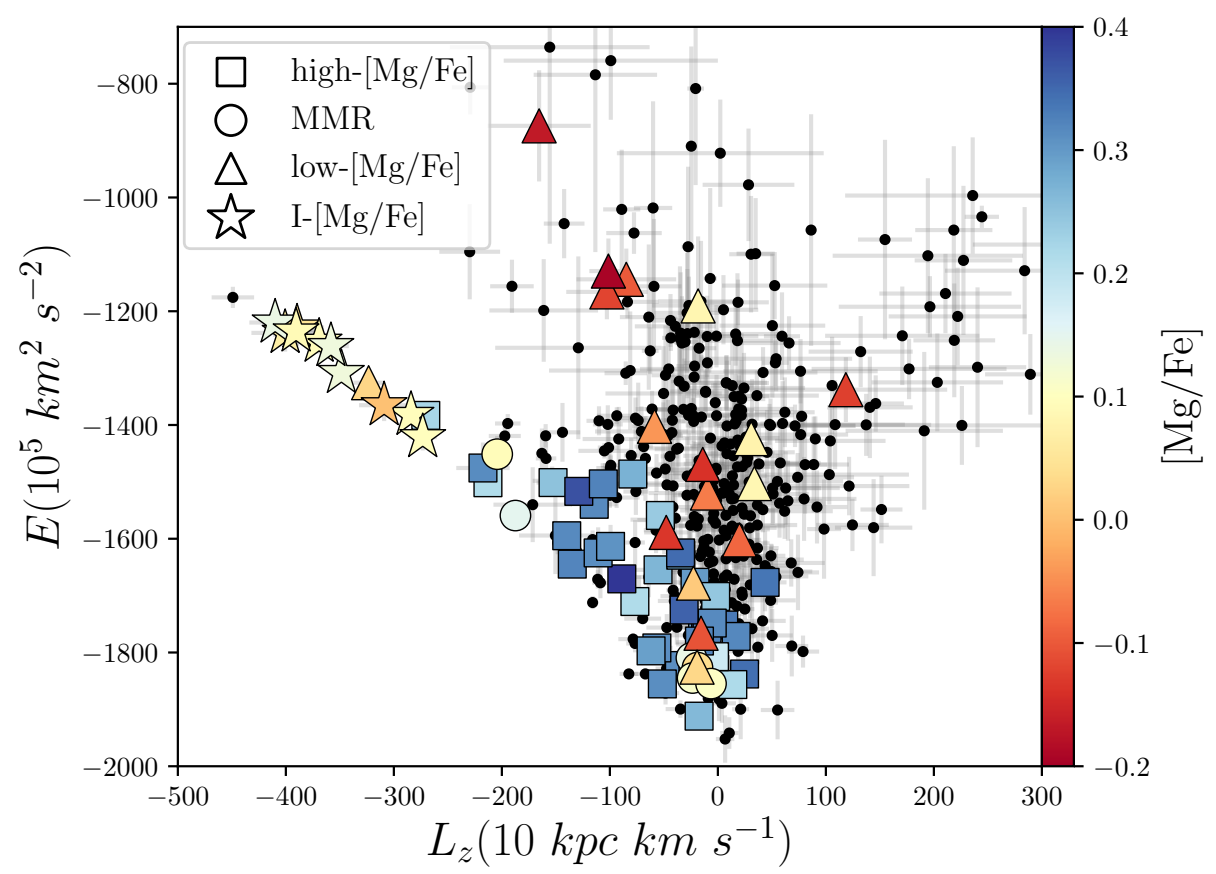

Figure 9. The total orbital energy $(E)$ as a function of the z-component of the angular momentum $\left(L_{z}\right)$, with colours indicating $[\mathrm{Mg} / \mathrm{Fe}]$ abundance ratios for stars at $[\mathrm{Fe} / \mathrm{H}]>-0.75$, and black dots referring to stars with $[\mathrm{Fe} / \mathrm{H}]<-0.75$.

$\left|z_{\max }\right| \sim 6-8 \mathrm{kpc}$ and $e \sim$ uniformly distributed over [0.4,1.0]; the latter, $r_{\text {apo }} \sim 30-40 \mathrm{kpc},\left|z_{\max }\right| \sim 18 \mathrm{kpc}$, and $e \sim 0.85$.

Our results are quite consistent with these values. However, our low- $[\mathrm{Mg} / \mathrm{Fe}]$ sample do not exhibit preferentially retrograde orbits. Besides, their chemical abundances, locations and velocities are consistent with the low- $[\mathrm{Mg} / \mathrm{Fe}]$ population at $[\mathrm{Fe} / \mathrm{H}]>-0.75$ being comprised by Sgr candidates.

Their chemical trends and their orbital parameter distributions resemble more the ones of metal-poor stars than those displayed by the high- $[\mathrm{Mg} / \mathrm{Fe}], \mathrm{MMR}$, or $\mathrm{I}-[\mathrm{Mg} / \mathrm{Fe}]$ groups. Thus, our low- $[\mathrm{Mg} / \mathrm{Fe}]$ group seems to be the metalrich tip of the metal-poor sample. However, the metal-poor group contains a larger fraction of stars on retrograde orbits.

Our metal-poor sample detected at high $|z|$ have properties more similar to the local metal-poor sample analysed by Helmi et al. (2018), Haywood et al. (2018), and Mackereth et al. (2018), based on the APOGEE DR14 database. They claimed that most of these stars could have been deposited by a single merger event. However, there should remain stars that have likely been accreted from other satellite galaxies, including Sgr, which would reach larger metallicities.

\subsection{The high- $[\mathrm{Mg} / \mathrm{Fe}]$ sequence: are they thick-disc stars?}

Haywood et al. (2018) obtained that stars (a subsample from APOGEE DR14 crossmatched with a high-quality Gaia data sample) sharing the red sequence in the HRD than the high$\alpha$, metal-rich $([\mathrm{Fe} / \mathrm{H}]>-0.8)$ Nissen \& Schuster population showed a considerable number of stars with $L_{z} \sim 0$ and even on retrograde orbits. Our analysis at high $|z|$ are in line with this. Interestingly, they also showed that chemically selected thick-disc stars share the same red sequence and the tail to rotation decreasing to zero, including even a few stars on retrograde orbits, pointing to a common origin with the high- $\alpha$ metal-rich Nissen \& Schuster stars.

We found that high- $[\mathrm{Mg} / \mathrm{Fe}]$ stars exhibit more-bound orbits that move closer to the Galactic plane than the low$[\mathrm{Mg} / \mathrm{Fe}]$ population. We also see that the $e$ distribution of the high- $[\mathrm{Mg} / \mathrm{Fe}]$ group is spread towards lower eccentricities, although the median value is $0.8 \pm 0.1$ (see Table 5). Ruchti et al. (2011) explored the variation of the eccentricity distribution for stars with $|z|$ between 1 and $3 \mathrm{kpc}$ from the Galactic plane. They show that the distribution peaks at low eccentricity, $\sim 0.4$, and the number of stars with the lowest eccentricities diminishes, whereas the number of stars with eccentricities larger than 0.8 increases as a function of $|z|$. The distribution of eccentricities displayed by our high$[\mathrm{Mg} / \mathrm{Fe}]$ stars differs from the Ruchti et al. distributions at lower $|z|$, as it peaks at high eccentricities, and the number of stars with $e=0.4$ is very low, but follows the logic of the increasing fraction of highly eccentric stars trend with $|z|$ showed in their work.

The high- $[\mathrm{Mg} / \mathrm{Fe}]$ chemical patterns are the same as for thick-disc stars. However, our stars are located at larger distances from the plane than previous works had characterized thick-disc members (e.g., Ruchti et al. 2011; Hayden et al. 2015). Additionally, our high- $[\mathrm{Mg} / \mathrm{Fe}]$ stars exhibit a lower average angular momentum than the classically attributed to this galactic component (e.g., Venn et al. 2004; Bensby et al. 2014). Some of the stars show even retrograde orbits.

However, Juric et al. (2008) showed that at $|z| \sim 5 \mathrm{kpc}$ from the plane the density of stars observed from Sloan data is well-reproduced by the sum of halo and thick-disc models with comparable densities. In addition, Schönrich \& Binney 


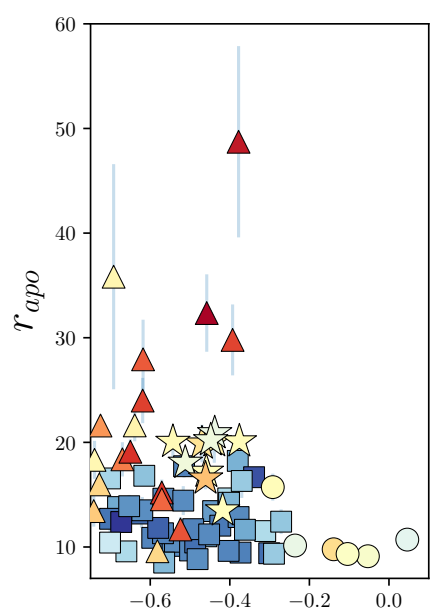

$[\mathrm{Fe} / \mathrm{H}]$

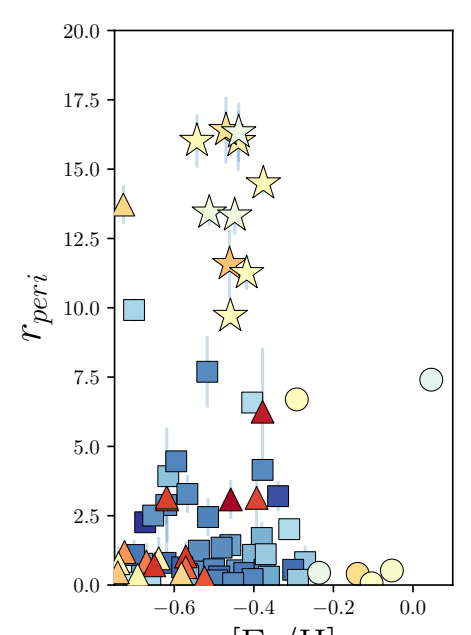

$\lceil\mathrm{Fe} / \mathrm{H}\rceil$
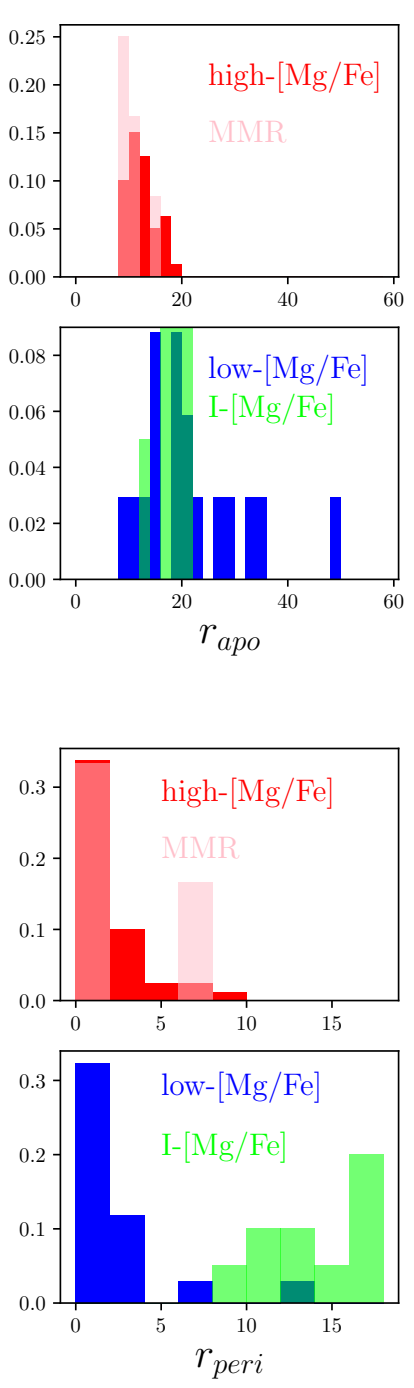

Figure 10. Left panels: Comparison of the maximum and minimum distance to the Galactic Centre, $r_{a p o}$ and $r_{p e r i}$, as a function of $[\mathrm{Fe} / \mathrm{H}]$, with colours indicating the $[\mathrm{Mg} / \mathrm{Fe}]$ abundances. Right panels: Distribution of $r_{a p o}$ and $r_{\text {peri }}$ for the high- $[\mathrm{Mg} / \mathrm{Fe}]$ and low$[\mathrm{Mg} / \mathrm{Fe}]$ populations selected as in Figure 7.

(2009) predicted from their model the existence in the Solar Neigbourhood of non-rotating thick-disc stars. If our high$[\mathrm{Mg} / \mathrm{Fe}]$ sample have born in the same cloud that formed the thick disc (as the similar chemistry suggests), we are detecting the tail toward no rotation (and even counter-rotation) at high distances from the plane.

Stars born in the disc may reach large $|z|$ in their orbits if they were somehow perturbed into more chaotic orbits. They can also reach large $|z|$ due to the interaction with the bar, when they move close to the Galactic centre, as explained in Moreno et al. (2014). However, this can only be the case for those stars showing low angular momenta, but large eccentricities. Those stars with low eccentricities could have not moved to such high $|z|$ in the absence of a significant merger event. If the high- $[\mathrm{Mg} / \mathrm{Fe}]$ stars in our sample originally formed as thick-disc stars, and now have

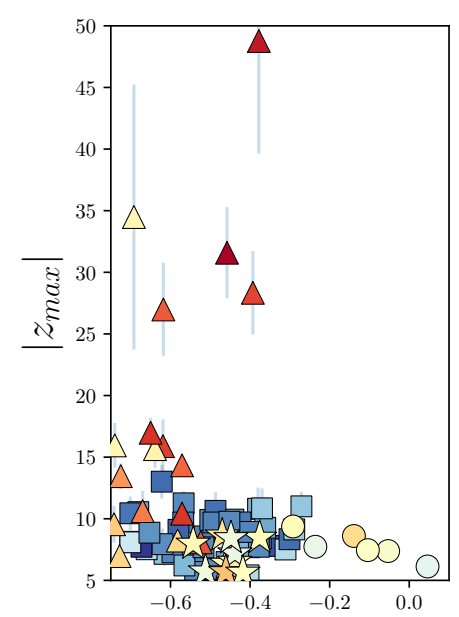

$[\mathrm{Fe} / \mathrm{H}\rceil$
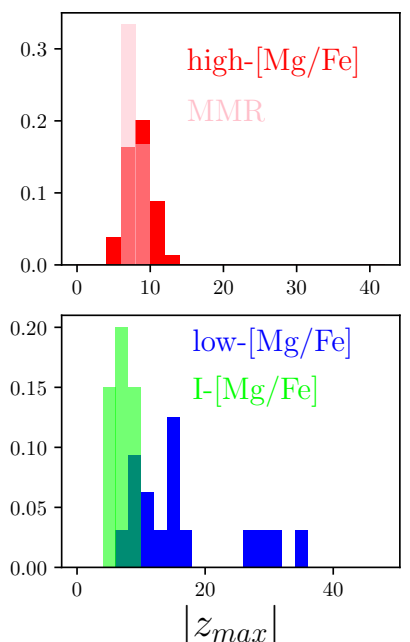

Figure 11. Left panels: Comparison of the maximum distance to the Galactic plane, $\left|z_{\max }\right|$, as a function of $[\mathrm{Fe} / \mathrm{H}]$, with colours indicating the $[\mathrm{Mg} / \mathrm{Fe}]$ abundances. Right panels: Distribution of $\left|z_{\max }\right|$ for the high-[Mg/Fe] and low-[Mg/Fe] populations selected as in Figure 7.

larger orbits and lower angular momentum, the merger had to be relatively massive in order to be able to change the stellar orbits so substantially (Qu et al. 2011; Jean-Baptiste et al. 2017).

\subsection{The I- $[\mathrm{Mg} / \mathrm{Fe}]$ stars: the discovery of a new sample of disc-heated stars.}

The $\mathrm{I}-[\mathrm{Mg} / \mathrm{Fe}]$ group is comprised by stars with $[\mathrm{Mg} / \mathrm{Fe}]$ $\sim+0.1$, and highly prograde motions with low eccentricity, reaching $\sim 8 \mathrm{kpc}$ from the Galactic plane, achieving relatively large $r_{a p o}$ and $r_{\text {peri }}$ during their orbits, and are significantly less bound to the Galaxy $(E \sim-1300)$.

The positions of these stars on the sky agree with those corresponding to the two stellar overdensities, Triangulum/Andromeda and A13, recently pointed out by Bergemann et al. (2018) and Hayes et al. (2018b). They found their samples located at distances from the Galactic center $\sim 18 \mathrm{kpc}$, which is consistent with our derived $r_{\text {peri }}$ and $r_{\text {apo }}$ results, $\sim 13$ and $20 \mathrm{kpc}$. We derived a median $V_{y}$ of $-28.0 \pm 13.7 \mathrm{~km} \mathrm{~s}^{-1}$, or $211.0 \pm 13.7 \mathrm{~km} \mathrm{~s}^{-1}$ if we convert it to the same reference frame as Bergemann et al. (2018), who derived a bit lower $V$ of $195 \pm 25 \mathrm{~km} \mathrm{~s}^{-1}$, consistent with our result within the error bars. Inspection of the chemical abundances showed that our sample is a little more metal rich $(<[\mathrm{Fe} / \mathrm{H}]>=-0.45 \pm 0.05)$ than Bergemann et al. $(<[\mathrm{Fe} / \mathrm{H}]>=-0.59 \pm 0.12)$ and Hayes et al. $(<[\mathrm{Fe} / \mathrm{H}]>=-0.8)$. Our $[\mathrm{X} / \mathrm{Fe}]$ ratios for $\mathrm{O}, \mathrm{Mg}$, and $\mathrm{Ti}$ are consistent with those derived by Bergemann et al., although theirs are a bit larger, likely due to the different methodology applied. The comparison of $[\mathrm{X} / \mathrm{Fe}]$ for $\mathrm{O}, \mathrm{Mg}$, $\mathrm{Ca}, \mathrm{K}, \mathrm{Mn}$, and Ni with those in Hayes et al. (which came from the same ASPCAP-analysed DR14 database) shows similar values than their sample of stars at slightly lower metallicities. 

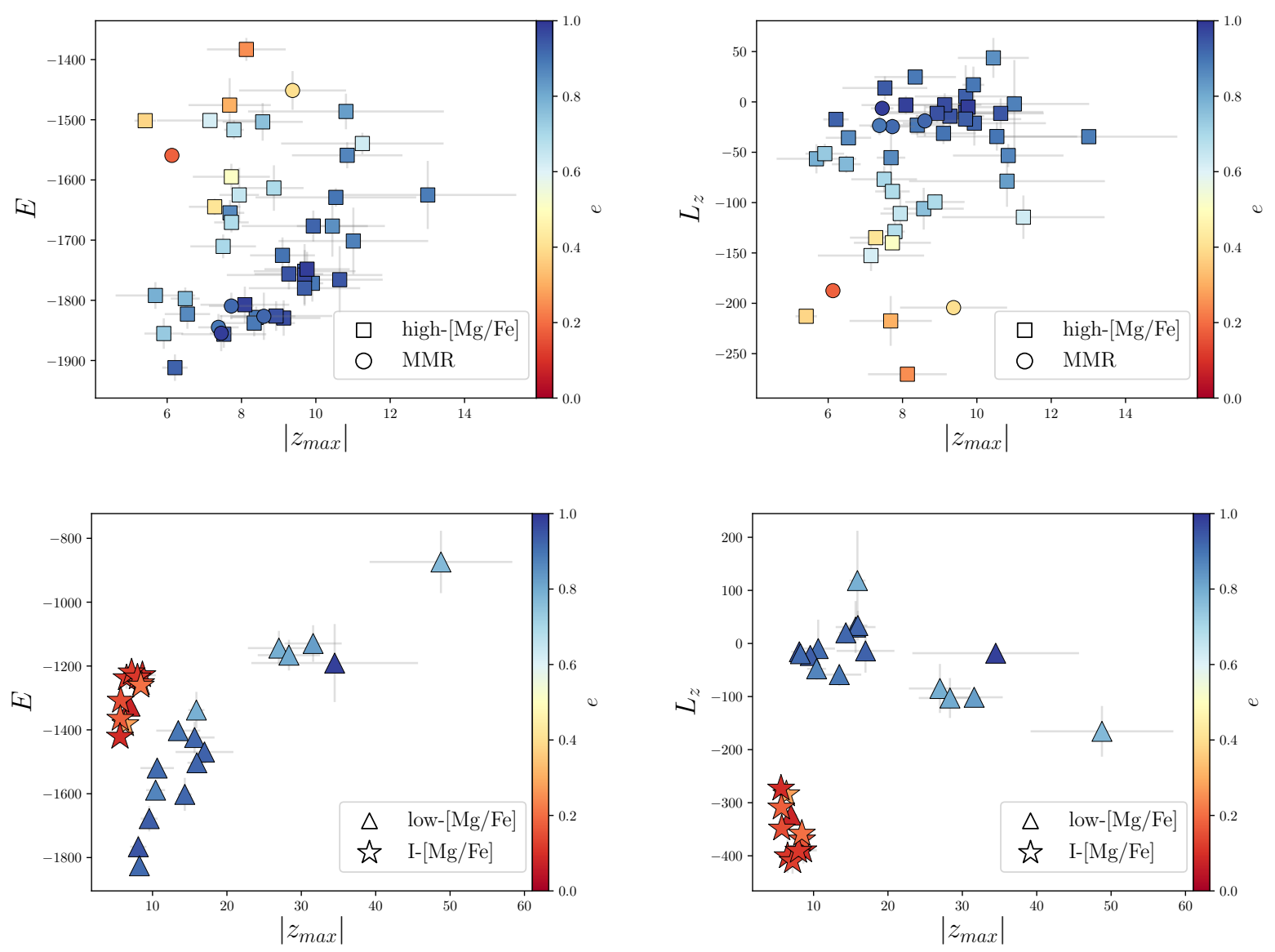

Figure 12. The total orbital energy (left panels) and the z-component of the angular momentum (right panels), as a function of the maximum vertical excursion from the Galactic plane $\left(\left|z_{\max }\right|\right)$ for the high- $[\mathrm{Mg} / \mathrm{Fe}]$ and MMR stars (top panels, dots and triangles, respectively) and for the low- $[\mathrm{Mg} / \mathrm{Fe}]$ and $\mathrm{I}-[\mathrm{Mg} / \mathrm{Fe}]$ (bottom panels, dots and triangles, respectively), with colours indicating the orbital eccentricity.

Both works have proposed that these stars were born in the disc but later heated into their actual orbits due to tidal effects from a merger. Our derived orbital parameters, in particular the large angular momentum, low eccentricities, relatively low $\left|z_{\max }\right|$ and large energies, are also consistent with disc orbits heated to larger heights from the plane. Their chemistry certainly points to formation in the Galactic outer disc. The sample of stars considered here have not been previously reported. Hence, our $\mathrm{I}-[\mathrm{Mg} / \mathrm{Fe}]$ group is a new sample of stars, likely belonging to these two stellar overdensities, and the orbital parameters derived from this analysis are compatible with the disc origin claimed in previous works. Table 3 report their APOGEE_ID as well as coordinates, radial velocities, stellar parameters and $[\mathrm{Fe} / \mathrm{H}]$.

\section{CONCLUSIONS}

We have analysed a sample of 504 halo candidate stars located at $|z|>5 \mathrm{kpc}$ from the Galactic plane, selected from the APOGEE DR14 database. We derive the MDF as a function of $|z|$, and detect a metal-rich tail up to $|z|>10 \mathrm{kpc}$. We perform a chemical analysis of these metal-rich stars using the elemental abundances derived by the ASPCAP pipeline, and a kinematical and dynamical analysis deriving orbits from the radial velocities also provided by the APOGEE DR14 database, spectroscopic distances from the StarHorse code, and high-quality proper motions from Gaia DR2.

Our main conclusions are the following:

(i) We detect metal-rich stars with $-0.75<[\mathrm{Fe} / \mathrm{H}]<0.0$ moving with halo-like kinematics $\left(V_{\text {tot }}>200 \mathrm{~km} / \mathrm{s}\right)$ at distances up to $|z|>10 \mathrm{kpc}$ from the Galactic plane. These stars show an $[\alpha / \mathrm{Fe}]$ vs. $[\mathrm{Fe} / \mathrm{H}]$ dichotomy: a high- $[\mathrm{Mg} / \mathrm{Fe}]$ group with a high constant $[\mathrm{Mg} / \mathrm{Fe}] \sim+0.3$ and a knee at $[\mathrm{Fe} / \mathrm{H}] \sim-0.4$; and a low- $[\mathrm{Mg} / \mathrm{Fe}]$ group with $[\mathrm{Mg} / \mathrm{Fe}]$ lower than +0.1 , following the decreasing $[\mathrm{Mg} / \mathrm{Fe}]$ trend with $[\mathrm{Fe} / \mathrm{H}]$ that dominates the bulk of halo stars at lower metallicities. The high- $[\mathrm{Mg} / \mathrm{Fe}]$ group shows a tail towards disclike $V_{\text {tot }}$.

(ii) We also detect a third chemically distinct group among metal-rich stars at high- $|z|$, the intermediate- $[\mathrm{Mg} / \mathrm{Fe}]$ stars, lying between the previous two sequences, with $[\mathrm{Mg} / \mathrm{Fe}] \sim+0.1$ and $[\mathrm{Fe} / \mathrm{H}] \sim-0.5$, but moving with disclike kinematics.

(iii) The high- $[\mathrm{Mg} / \mathrm{Fe}]$, low- $[\mathrm{Mg} / \mathrm{Fe}]$, and I- $[\mathrm{Mg} / \mathrm{Fe}]$ stellar groups are also chemically distinct in other elemental species (O, Si, S, Ca, Ti, Ni, and K). 
(iv) The chemical distinct stellar groups are also kinematically and dynamically different.

(v) The high- $[\mathrm{Mg} / \mathrm{Fe}]$ stars exhibit a distribution of $V_{y}$ with primarily slightly prograde rotation, the angular momentum decreasing to 0 as $\left|z_{\max }\right|$ increases. Their orbits are more bound to the Galaxy and closer to the Galactic plane than the low- $[\mathrm{Mg} / \mathrm{Fe}]$ stars. They typically exhibit large eccentricities, $e=0.8$, although the distribution extends towards lower values for a few stars. Their chemical abundances are similar to that displayed by thick-disc stars. Our results point to the high- $[\mathrm{Mg} / \mathrm{Fe}]$ group as a thick-disc tail towards $L_{z} \sim 0$ at large $|z|$.

(vi) The low- $[\mathrm{Mg} / \mathrm{Fe}]$ group has a $V_{y}$ distribution centreed around zero net rotation, although slightly skewed toward prograde motions. Their orbits are less bound and reach larger $\left|z_{\max }\right|$ and $r_{a p o}$. Their chemical abundances, location and velocities are compatible with an origin in the Sagittarius dwarf satellite.

(vii) The $\mathrm{I}-[\mathrm{Mg} / \mathrm{Fe}]$ stars exhibit the largest prograde motion of the metal-rich halo sample, and move on loweccentricity $(e \sim 0.1)$ orbits that are relatively close to the Galactic plane. However, they are significantly less bound to the Galaxy, and reach large $r_{a p o}$ and $r_{p e r i}$ during their orbits. The I- $[\mathrm{Mg} / \mathrm{Fe}]$ stars very likely belong to the two stellar overdensities, Triangulum-Andromeda and A13, comprising a new sample of candidates not prevously reported.

We believe that the most likely explanation for the observed properties of the stars in our sample is that a fraction of them arose due to influence of a relatively massive accretion event that had a strong interaction with a nascent thick disc, as has been suggested by a number of recent studies. Clearly, more detailed comparisons with numerical simulations would prove illuminating.

\section{ACKNOWLEDGEMENTS}

We thank the anonymous referee for the comments provided which considerably improved this manuscript. E.F.A. would like to thank Edouard Bernard, Govind Nandakumar, Álvaro Rojas-Arriagada and Georges Kordopatis for very useful suggestions and discussions. E.F.A. and A.R.B. acknowledge financial support from the ANR 14-CE33-014-01. E.F.A. also thanks for partial support provided by CONACyT of México (grant 241732). J.G.F-T is supported by FONDECYTNo. 3180210. L.C. thanks for the financial supports provided by CONACyT of México (grant 241732), by PAPIIT of México (IA101217, IA101517) and by MINECO of Spain (AYA2015-65205-P). T.C.B. acknowledges partial support for this work from grant PHY 14-30152; Physics Frontier Center/ JINA Center for the Evolution of the Elements (JINA-CEE), awarded by the US National Science Foundation. OZ and DAGH acknowledge support provided by the Spanish Ministry of Economy and Competitiveness (MINECO) under grant AYA-2017-88254-P. EM and APV acknowledge the grants DGAPA PAPIIT IN105916 and G100319. APV acknowledges FAPESP for the postdoctoral fellowship 2017/15893-1.

Funding for the GravPot16 software has been provided by the Centre national d'etudes spatiales (CNES) through grant 0101973 and UTINAM Institute of the Université de Franche-Comté, supported by the Région de Franche-Comté and Institut des Sciences de l'Univers (INSU). Simulations have been executed on computers from the Utinam Institute of the Université de Franche- Comté, supported by the Région de Franche-Comté and Institut des Sciences de l'Univers (INSU), and on the supercomputer facilities of the Mésocentre de calcul de Franche-Comté.

This work has made use of data from the European Space Agency (ESA) mission Gaia (https://www.cosmos. esa.int/gaia), processed by the Gaia Data Processing and Analysis Consortium (DPAC, https://www.cosmos.esa. int/web/gaia/dpac/consortium). Funding for the DPAC has been provided by national institutions, in particular the institutions participating in the Gaia Multilateral Agreement.

Funding for the Sloan Digital Sky Survey IV has been provided by the Alfred P. Sloan Foundation, the U.S. Department of Energy Office of Science, and the Participating Institutions. SDSS acknowledges support and resources from the Center for High-Performance Computing at the University of Utah. The SDSS web site is www.sdss.org.

SDSS is managed by the Astrophysical Research Consortium for the Participating Institutions of the SDSS Collaboration including the Brazilian Participation Group, the Carnegie Institution for Science, Carnegie Mellon University, the Chilean Participation Group, the French Participation Group, Harvard-Smithsonian Center for Astrophysics, Instituto de Astrofísica de Canarias, The Johns Hopkins University, Kavli Institute for the Physics and Mathematics of the Universe (IPMU) / University of Tokyo, the Korean Participation Group, Lawrence Berkeley National Laboratory, Leibniz Institut für Astrophysik Potsdam (AIP), MaxPlanck-Institut fãijr Astronomie (MPIA Heidelberg), MaxPlanck-Institut fãijr Astrophysik (MPA Garching), MaxPlanck-Institut fãijr Extraterrestrische Physik (MPE), National Astronomical Observatories of China, New Mexico State University, New York University, University of Notre Dame, Observatório Nacional / MCTI, The Ohio State University, Pennsylvania State University, Shanghai Astronomical Observatory, United Kingdom Participation Group, Universidad Nacional Autónoma de México, University of Arizona, University of Colorado Boulder, University of Oxford, University of Portsmouth, University of Utah, University of Virginia, University of Washington, University of Wisconsin, Vanderbilt University, and Yale University.

\section{REFERENCES}

Ablimit, I., \& Zhao, G. 2018, ApJ, 855, 126

Abolfathi, B., Aguado, D. S., Aguilar, G., et al. 2018, ApJS, 235, 42

Albareti, F. D., Allende Prieto, C., Almeida, A., et al. 2017, ApJS, 233,25

Allen, C., \& Santillan, A. 1991, Rev. Mex. Astron. Astrofis., 22, 255

Allende Prieto, C., Fernández-Alvar, E., Schlesinger, K. J., et al. 2014, A\&A, 568, A7

Amôres, E. B., Robin, A. C., \& Reylé, C. 2017, A\&A, 602, A67

An, D., Beers, T. C., Santucci, R. M., et al. 2015, ApJ, 813, L28

Anders, F., Chiappini, C., Santiago, B. X., et al. 2014, A\&A, 564, A115

Bailer-Jones, C. A. L., Rybizki, J., Fouesneau, M., Mantelet, G., \& Andrae, R. 2018, AJ, 156, 58 
Beers, T. C., Carollo, D., Ivezić, Ž., et al. 2012, ApJ, 746, 34

Belokurov, V., Erkal, D., Evans, N. W., Koposov, S. E., \& Deason, A. J. 2018, MNRAS, 478, 611

Bensby, T., Feltzing, S., \& Oey, M. S. 2014, A\&A, 562, A71

Bergemann, M., Collet, R., Schönrich, R., et al. 2017, ApJ, 847, 16

Bergemann, M., Sesar, B., Cohen, J. G., et al. 2018, Nature, 555, 334

Bernard, E. J., Ferguson, A. M. N., Schlafly, E. F., et al. 2016, MNRAS, 463, 1759

Binney, J., Burnett, B., Kordopatis, G., et al. 2014, MNRAS, 437, 351

Bland-Hawthorn, J., \& Gerhard, O. 2016, ARA\&A, 54, 529

Blanton, M. R., Bershady, M. A., Abolfathi, B., et al. 2017, AJ, 154,28

Bonaca, A., Conroy, C., Wetzel, A., Hopkins, P. F., \& Kereš, D. 2017, ApJ, 845, 101

Bressan, A., Marigo, P., Girardi, L., et al. 2012, MNRAS, 427, 127

Brunthaler, A., Reid, M. J., Menten, K. M., et al. 2011, Astronomische Nachrichten, 332, 461

Carlin, J. L., Sheffield, A. A., Cunha, K., \& Smith, V. V. 2018, arXiv:1805.04120

Carollo, D., Beers, T. C., Lee, Y. S., et al. 2007, Nature, 450, 1020

Carollo, D., Beers, T. C., Chiba, M., et al. 2010, ApJ, 712, 692

Contreras Ramos, R., Minniti, D., Fernandez-Trincado, J. G., et al. 2018, arXiv:1807.02065

Deason, A. J., Belokurov, V., \& Evans, N. W. 2011, MNRAS, 416, 2903

Deason, A. J., Belokurov, V., Koposov, S. E., \& Lancaster, L. 2018, ApJ, 862, L1

Eggen, O. J., Lynden-Bell, D., \& Sandage, A. R. 1962, ApJ, 136, 748

Einasto, J. 1979, The Large-Scale Characteristics of the Galaxy, 84,451

Eisenstein, D. J., Weinberg, D. H., Agol, E., et al. 2011, AJ, 142, 72

Fernández-Alvar, E., Allende Prieto, C., Schlesinger, K. J., et al. 2015, A\&A, 577, A81

Fernández-Alvar, E., Carigi, L., Allende Prieto, C., et al. 2017, MNRAS, 465, 1586

Fernández-Alvar, E., Carigi, L., Schuster, W. J., et al. 2018, ApJ, 852,50

Fernández-Trincado, J. G., Robin, A. C., Moreno, E., et al. 2016, ApJ, 833, 132

Fernández-Trincado, J. G., Zamora, O., García-Hernández, D. A., et al. 2017, ApJ, 846, L2

Fernández-Trincado, J. G., Beers, T. C., Placco, V. M., et al. 2019, arXiv:1904.05884

Font, A. S., McCarthy, I. G., Crain, R. A., et al. 2011, MNRAS, 416, 2802

Fuhrmann, K. 1999, Ap\&SS, 265, 265

Gaia Collaboration, Prusti, T., de Bruijne, J. H. J., et al. 2016, A\&A, 595, A1

Gaia Collaboration, Brown, A. G. A., Vallenari, A., et al. 2018, A\&A, 616, A1

Gaia Collaboration, Babusiaux, C., van Leeuwen, F., et al. 2018, A\&A, 616, A10

García Pérez, A. E., Allende Prieto, C., Holtzman, J. A., et al. 2016, AJ, 151, 144

Girardi, L., Barbieri, M., Groenewegen, M. A. T., et al. 2012, Astrophysics and Space Science Proceedings, 26, 165

Grillmair, C. J. 2009, ApJ, 693, 1118

Gunn, J. E., Siegmund, W. A., Mannery, E. J., et al. 2006, AJ, 131,2332

Hasselquist, S., Shetrone, M., Smith, V., et al. 2017, ApJ, 845, 162

Hawkins, K., Jofré, P., Masseron, T., \& Gilmore, G. 2015, MN-
RAS, 453, 758

Hawkins, K., Masseron, T., Jofré, P., et al. 2016, A\&A, 594, A43

Hayden, M. R., Bovy, J., Holtzman, J. A., et al. 2015, ApJ, 808, 132

Hayes, C. R., Majewski, S. R., Shetrone, M., et al. 2018, ApJ, 852,49

Hayes, C. R., Majewski, S. R., Hasselquist, S., et al. 2018, ApJ, $859, \mathrm{~L} 8$

Haywood, M., Di Matteo, P., Lehnert, M. D., et al. 2018, ApJ, 863,113

Helmi, A., Babusiaux, C., Koppelman, H. H., et al. 2018, arXiv:1806.06038

Holtzman, J. A., Shetrone, M., Johnson, J. A., et al. 2015, AJ, 150,148

Holtzman, J. A., Hasselquist, S., Shetrone, M., et al. 2018, AJ, 156,125

Jönsson, H., Allende Prieto, C., Holtzman, J. A., et al. 2018, AJ, 156,126

Jean-Baptiste, I., Di Matteo, P., Haywood, M., et al. 2017, A\&A, 604, A106

Jurić, M., Ivezić, Ž., Brooks, A., et al. 2008, ApJ, 673, 864

Lancaster, L., Koposov, S. E., Belokurov, V., Evans, N. W., \& Deason, A. J. 2018, arXiv:1807.04290

Lee, Y. S., Beers, T. C., Kim, Y. K., et al. 2017, ApJ, 836, 91

Leung, H. W., \& Bovy, J. 2019, arXiv:1902.08634

Li, T. S., Sheffield, A. A., Johnston, K. V., et al. 2017, ApJ, 844, 74

Libralato, M., Bellini, A., Bedin, L. R., et al. 2018, ApJ, 854, 45 Majewski, S. R., Schiavon, R. P., Frinchaboy, P. M., et al. 2017, AJ, 154, 94

Mackereth, J. T., Schiavon, R. P., Pfeffer, J., et al. 2019, MNRAS, 482,3426

Martin, N. F., Ibata, R. A., \& Irwin, M. 2007, ApJ, 668, L123

Mateu, C., \& Vivas, A. K. 2018, MNRAS, 479, 211

McMillan, P. J. 2018, Research Notes of the American Astronomical Society, 2, 51

Michalik, D., Lindegren, L., \& Hobbs, D. 2015, A\&A, 574, A115

Moreno, E., Pichardo, B., \& Velázquez, H. 2014, ApJ, 793, 110

Myeong, G. C., Evans, N. W., Belokurov, V., Sanders, J. L., \& Koposov, S. E. 2018, ApJ, 856, L26

Myeong, G. C., Evans, N. W., Belokurov, V., Sanders, J. L., \& Koposov, S. E. 2018, ApJ, 863, L28

ewberg H. J., Carlin J. L. 2015, Tidal Streams in the Local Group and Beyond, Springer, New York

Nidever, D. L., Holtzman, J. A., Allende Prieto, C., et al. 2015, AJ, 150, 173

Nissen, P. E. 2004, Origin and Evolution of the Elements, 154

Nissen, P. E., \& Schuster, W. J. 2010, A\&A, 511, L10

Nissen, P. E., \& Schuster, W. J. 2011, A\&A, 530, A15

Pichardo, B., Martos, M., \& Moreno, E. 2004, ApJ, 609, 144

Perottoni, H. D., Rocha-Pinto, H. J., Girardi, L., et al. 2018, MNRAS, 473, 1461

Price-Whelan, A. M., Johnston, K. V., Sheffield, A. A., Laporte, C. F. P., \& Sesar, B. 2015, MNRAS, 452, 676

Qu, Y., Di Matteo, P., Lehnert, M. D., van Driel, W., \& Jog, C. J. 2011, A\&A, 535, A5

Queiroz, A. B. A., Anders, F., Santiago, B. X., et al. 2018, MNRAS, 476, 2556

Recio-Blanco, A., de Laverny, P., Kordopatis, G., et al. 2014, A\&A, 567, A5

Recio-Blanco, A., Rojas-Arriagada, A., de Laverny, P., et al. 2017, A\&A, 602, L14

Robin, A. C., Reylé, C., Fliri, J., et al. 2014, A\&A, 569, A13

Rocha-Pinto, H. J., Majewski, S. R., Skrutskie, M. F., Crane, J. D., \& Patterson, R. J. 2004, ApJ, 615, 732

Ruchti, G. R., Fulbright, J. P., Wyse, R. F. G., et al. 2011, ApJ, 737,9 
Santiago, B. X., Brauer, D. E., Anders, F., et al. 2016, A\&A, 585, A42

Schiappacasse-Ulloa, J., Tang, B., Fernández-Trincado, J. G., et al. 2018, arXiv:1806.09575

Schönrich, R., \& Binney, J. 2009, MNRAS, 399, 1145

Schönrich, R., Binney, J., \& Dehnen, W. 2010, MNRAS, 403, 1829

Schönrich, R., Asplund, M., \& Casagrande, L. 2014, ApJ, 786, 7

Schuster, W. J., Moreno, E., Nissen, P. E., \& Pichardo, B. 2012, A\&A, 538, A21

Sharma, S., Johnston, K. V., Majewski, S. R., et al. 2010, ApJ, 722,750

Simion, I. T., Belokurov, V., \& Koposov, S. E. 2019, MNRAS, 482,921

Soubiran, C., Bienaymé, O., \& Siebert, A. 2003, A\&A, 398, 141

Tang, B., Fernández-Trincado, J. G., Geisler, D., et al. 2018, ApJ, 855,38

Tissera, P. B., White, S. D. M., \& Scannapieco, C. 2012, MNRAS, 420,255

Tissera, P. B., Scannapieco, C., Beers, T. C., \& Carollo, D. 2013, MNRAS, 432, 3391

Tissera, P. B., Beers, T. C., Carollo, D., \& Scannapieco, C. 2014, MNRAS, 439, 3128

Venn, K. A., Irwin, M., Shetrone, M. D., et al. 2004, AJ, 128, 1177

Wang, H.-F., Liu, C., Xu, Y., Wan, J.-C., \& Deng, L. 2018, MNRAS, 478, 3367

Wilson, J. C., Hearty, F., Skrutskie, M. F., et al. 2010, Proc. SPIE, $7735,77351 \mathrm{C}$

Zhang, J., Shi, J., Pan, K., Allende Prieto, C., \& Liu, C. 2017, ApJ, 835, 90

Zasowski, G., Johnson, J. A., Frinchaboy, P. M., et al. 2013, AJ, 146,81

Zasowski, G., Cohen, R. E., Chojnowski, S. D., et al. 2017, AJ, 154,198

Zasowski, G., Schultheis, M., Hasselquist, S., et al. 2018, arXiv:1811.01097

Zinn, J. C., Pinsonneault, M. H., Huber, D., \& Stello, D. 2018, arXiv:1805.02650

This paper has been typeset from a $\mathrm{T}_{\mathrm{E}} \mathrm{X} / \mathrm{LAT}_{\mathrm{E}} \mathrm{X}$ file prepared by the author. 
Table 1. Stellar parameters, $[\mathrm{Fe} / \mathrm{H}]$ and $[\mathrm{Mg} / \mathrm{Fe}]$ abundances for our 504 halo candidates. A complete version of this table is available online.

\begin{tabular}{lcccccccc}
\hline \hline APOGEE ID & $\mathrm{ra}$ & $\mathrm{dec}$ & $\mathrm{l}$ & $\mathrm{b}$ & $T_{\text {eff }}(K)$ & $\log g$ & {$[\mathrm{Fe} / \mathrm{H}]$} & {$[\mathrm{Mg} / \mathrm{Fe}]$} \\
2M13381056+3823163 & 204.544 & 38.388 & 84.860 & 75.082 & $4021.1 \pm 47.3$ & $1.36 \pm 0.06$ & $-0.38 \pm 0.01$ & $0.32 \pm 0.02$ \\
2M12472244-0140359 & 191.843 & -1.677 & 300.825 & 61.178 & $4466.1 \pm 62.5$ & $1.69 \pm 0.07$ & $-0.72 \pm 0.01$ & $-0.04 \pm 0.03$ \\
2M14202222+5252230 & 215.093 & 52.873 & 96.269 & 59.407 & $4347.5 \pm 57.6$ & $1.52 \pm 0.06$ & $-0.29 \pm 0.01$ & $0.09 \pm 0.02$ \\
2M16264146+2649592 & 246.673 & 26.833 & 45.571 & 42.432 & $4109.9 \pm 50.3$ & $1.10 \pm 0.06$ & $-0.62 \pm 0.01$ & $0.24 \pm 0.03$ \\
2M10085156+4430006 & 152.215 & 44.500 & 173.871 & 53.250 & $4075.0 \pm 48.4$ & $1.29 \pm 0.05$ & $-0.06 \pm 0.01$ & $0.17 \pm 0.02$ \\
2M12161853+1452490 & 184.077 & 14.880 & 267.446 & 75.274 & $4268.9 \pm 55.2$ & $1.44 \pm 0.06$ & $-0.52 \pm 0.01$ & $-0.10 \pm 0.03$ \\
$\cdots$ & & & & & & & \\
$\cdots$
\end{tabular}

Table 2. Radial velocities, parallaxes, proper motions, and distances for our 504 halo candidates. A complete version of this table is available online.

\begin{tabular}{lccccc}
\hline \hline APOGEE ID & $v_{\text {rad }}\left(\mathrm{km} \mathrm{s}^{-1}\right)$ & $\pi($ mas $)$ & $\mu_{\text {ra }}\left(\right.$ mas $\left._{y r}{ }^{-1}\right)$ & $\mu_{\text {dec }}\left(\right.$ mas $\left._{y r}{ }^{-1}\right)$ & $d_{\text {sun }}($ pc $)$ \\
2M13381056+3823163 & $-90.13 \pm 0.08$ & $0.0979 \pm 0.0222$ & $1.125 \pm 0.018$ & $-4.451 \pm 0.029$ & $6157.7 \pm 606.8$ \\
2M12472244-0140359 & $-66.74 \pm 0.17$ & $0.118 \pm 0.026$ & $3.766 \pm 0.054$ & $-8.633 \pm 0.030$ & $5734.2 \pm 746.0$ \\
2M14202222+5252230 & $-29.02 \pm 0.06$ & $0.1013 \pm 0.0201$ & $2.284 \pm 0.029$ & $-7.050 \pm 0.035$ & $5975.7 \pm 1317.2$ \\
2M16264146+2649592 & $1.33 \pm 0.01$ & $0.131 \pm 0.023$ & $-2.848 \pm 0.034$ & $-2.704 \pm 0.039$ & $7533.3 \pm 663.7$ \\
2M10085156+4430006 & $5.467 \pm 0.002$ & $0.227 \pm 0.049$ & $-1.715 \pm 0.055$ & $-2.734 \pm 0.071$ & $7536.5 \pm 690.6$ \\
2M12161853+1452490 & $111.80 \pm 0.20$ & $0.0934 \pm 0.0507$ & $-5.598 \pm 0.0900$ & $-5.704 \pm 0.086$ & $6486.6 \pm 618.7$ \\
$\cdots$ & & & & & \\
\hline \hline
\end{tabular}

Table 3. I- $[\mathrm{Mg} / \mathrm{Fe}]$ stars identified as belonging to the Triangulum/Andromeda and A13 stellar overdensities.

\begin{tabular}{lcccccc}
\hline \hline APOGEE ID & $\mathrm{ra}$ & $\mathrm{dec}$ & $v_{\text {rad }}\left(\mathrm{km} \mathrm{s}^{-1}\right)$ & $T_{\text {eff }}(\mathrm{K})$ & $\log g$ & {$[\mathrm{Fe} / \mathrm{H}]$} \\
2M03255238+0921586 & 51.468 & 9.366 & $27.46 \pm 0.17$ & $4532.8 \pm 76.5$ & $2.07 \pm 0.07$ & $-0.46 \pm 0.01$ \\
2M07421181+6645232 & 115.549 & 66.756 & $-49.98 \pm 0.11$ & $4412.3 \pm 78.8$ & $1.60 \pm 0.08$ & $-0.44 \pm 0.01$ \\
2M07510819+5232100 & 117.784 & 52.536 & $-24.26 \pm 0.00$ & $4238.6 \pm 63.6$ & $1.32 \pm 0.07$ & $-0.47 \pm 0.01$ \\
2M08000496+5223185 & 120.0207 & 52.388 & $8.49 \pm 0.00$ & $4071.7 \pm 55.3$ & $1.09 \pm 0.06$ & $-0.38 \pm 0.01$ \\
2M08031715+4305211 & 120.821 & 43.089 & $-38.92 \pm 0.11$ & $4259.6 \pm 61.3$ & $1.37 \pm 0.07$ & $-0.44 \pm 0.01$ \\
2M08170874+3252000 & 124.286 & 32.867 & $54.83 \pm 0.21$ & $4592.5 \pm 76.8$ & $2.01 \pm 0.07$ & $-0.54 \pm 0.01$ \\
2M08455950+1141225 & 131.498 & 11.690 & $69.44 \pm 0.07$ & $4089.4 \pm 49.5$ & $1.11 \pm 0.06$ & $-0.51 \pm 0.01$ \\
2M09083889+4130457 & 137.162 & 41.513 & $-59.24 \pm 0.08$ & $4275.6 \pm 65.1$ & $1.45 \pm 0.07$ & $-0.45 \pm 0.01$ \\
2M09233417-0657105 & 140.892 & -6.953 & $115.94 \pm 0.11$ & $4290.2 \pm 62.3$ & $1.44 \pm 0.07$ & $-0.46 \pm 0.01$ \\
2M10144610+0233578 & 153.692 & 2.566 & $53.07 \pm 0.00$ & $4556.5 \pm 78.4$ & $1.85 \pm 0.07$ & $-0.42 \pm 0.01$ \\
\hline \hline
\end{tabular}


Table 4. Median and median absolute deviation (MAD) of the Galactocentric velocity components for the metal-rich halo stars, separated into four groups, as explained in the text - high-[Mg/Fe], most metal-rich (MMR), low-[Mg/Fe], and intermediate- $\mathrm{Mg}(\mathrm{I}-[\mathrm{Mg} / \mathrm{Fe}])$.

\begin{tabular}{|c|c|c|c|c|c|c|}
\hline & \multicolumn{3}{|c|}{$V_{y}\left(k m s^{-1}\right)$} & \multicolumn{3}{|c|}{$\sqrt{V_{x}^{2}+V_{z}^{2}}\left(k m s^{-1}\right)$} \\
\hline & all & $d_{B J}<40 \%$ & $\pi_{e}<50 \%$ & all & $d_{B J}<40 \%$ & $\pi_{e}<50 \%$ \\
\hline \multicolumn{7}{|c|}{ high- $[\mathrm{Mg} / \mathrm{Fe}]$} \\
\hline median & -207.8 & -209.7 & -171.9 & 95.6 & 100.1 & 86.2 \\
\hline MAD & 50.1 & 48.1 & 51.5 & 38.6 & 40.8 & 33.6 \\
\hline \multicolumn{7}{|l|}{ MMR } \\
\hline median & -201.1 & -199.8 & -201.1 & 102.1 & 103.5 & 102.1 \\
\hline MAD & 18.4 & 14.2 & 27.8 & 57.4 & 33.6 & 49.0 \\
\hline \multicolumn{7}{|c|}{ low- $[\mathrm{Mg} / \mathrm{Fe}]$} \\
\hline median & -239.6 & -228.9 & -242.4 & 115.8 & 133.2 & 101.2 \\
\hline MAD & 36.0 & 72.1 & 27.7 & 58.6 & 85.4 & 58.6 \\
\hline \multicolumn{7}{|c|}{$\mathrm{I}-[\mathrm{Mg} / \mathrm{Fe}]$} \\
\hline median & -28.0 & -16.8 & -33.5 & 61.9 & 59.6 & 45.8 \\
\hline MAD & 13.7 & 5.7 & 15.4 & 9.9 & 9.5 & 7.3 \\
\hline
\end{tabular}

Table 5. Median and median absolute deviation (MAD) of the orbital parameters $E, L_{z}$, and $e$ for the metal-rich halo stars, separated into four groups, as explained in the text - high- $[\mathrm{Mg} / \mathrm{Fe}]$, most metal-rich $(\mathrm{MMR})$, low- $[\mathrm{Mg} / \mathrm{Fe}]$, and intermediate- $[\mathrm{Mg} / \mathrm{Fe}](\mathrm{I}-[\mathrm{Mg} / \mathrm{Fe}])$.

\begin{tabular}{|c|c|c|c|c|c|c|c|c|c|}
\hline & \multicolumn{3}{|c|}{$E\left(10^{5} \mathrm{~km}^{2} \mathrm{~s}^{-2}\right)$} & \multicolumn{3}{|c|}{$L_{z}\left(10 k p c k m s^{-1}\right)$} & \multicolumn{3}{|c|}{$e$} \\
\hline & all & $d_{B J}<40 \%$ & $\pi_{e}<50 \%$ & all & $d_{B J}<40 \%$ & $\pi_{e}<50 \%$ & all & $d_{B J}<40 \%$ & $\pi_{e}<50 \%$ \\
\hline \multicolumn{10}{|c|}{ high- $[\mathrm{Mg} / \mathrm{Fe}]$} \\
\hline median & -1736.8 & -1752.3 & -1781.9 & -43.5 & -35.1 & -52.3 & 0.8 & 0.8 & 0.8 \\
\hline MAD & 91.9 & 76.1 & 82.4 & 40.1 & 28.8 & 43.4 & 0.1 & 0.1 & 0.1 \\
\hline \multicolumn{10}{|l|}{ MMR } \\
\hline median & -1809.3 & -1818.0 & -1790.6 & -23.4 & -24.0 & -18.9 & 0.9 & 0.9 & 0.9 \\
\hline MAD & 45.2 & 27.1 & 89.1 & 21.4 & 19.5 & 30.0 & 0.1 & 0.1 & 0.1 \\
\hline \multicolumn{10}{|c|}{ low-[Mg/Fe] } \\
\hline median & -1469.3 & -1545.9 & -1511.3 & -19.3 & -21.0 & -14.4 & 0.9 & 0.9 & 0.9 \\
\hline MAD & 132.7 & 181.9 & 99.8 & 39.3 & 16.1 & 39.3 & 0.03 & 0.03 & 0.01 \\
\hline \multicolumn{10}{|c|}{$\mathrm{I}-[\mathrm{Mg} / \mathrm{Fe}]$} \\
\hline median & -1261.2 & -1249.6 & -1334.7 & -358.3 & -379.5 & -323.6 & 0.1 & 0.1 & 0.2 \\
\hline MAD & 41.3 & 20.7 & 36.8 & 42.4 & 25.8 & 32.2 & 0.03 & 0.02 & 0.1 \\
\hline
\end{tabular}


Table 6. Median and median absolute deviation (MAD) of the orbital parameters $\left|z_{\max }\right|, r_{a p o}$, and $r_{\text {peri }}$ for the metal-rich halo stars, separated into four groups, as explained in the text - high- $[\mathrm{Mg} / \mathrm{Fe}]$, most metal-rich (MMR), low- $[\mathrm{Mg} / \mathrm{Fe}]$, and intermediate-[Mg/Fe] $(\mathrm{I}-[\mathrm{Mg} / \mathrm{Fe}])$.

\begin{tabular}{|c|c|c|c|c|c|c|c|c|c|}
\hline & & $\left|z_{\max }\right|(k p c)$ & & & $r_{a p o}(k p c)$ & & & $r_{p e r i}(k p c)$ & \\
\hline & all & $d_{B J}<40 \%$ & $\pi_{e}<50 \%$ & all & $d_{B J}<40 \%$ & $\pi_{e}<50 \%$ & all & $d_{B J}<40 \%$ & $\pi_{e}<50 \%$ \\
\hline \multicolumn{10}{|c|}{ high- $[\mathrm{Mg} / \mathrm{Fe}]$} \\
\hline median & 7.8 & 8.5 & 7.5 & 11.4 & 11.7 & 10.7 & 0.94 & 0.94 & 1.2 \\
\hline MAD & 1.5 & 1.3 & 1.2 & 2.0 & 1.9 & 1.7 & 0.70 & 0.68 & 0.92 \\
\hline \multicolumn{10}{|l|}{ MMR } \\
\hline median & 7.4 & 7.6 & 8.6 & 9.8 & 9.6 & 9.8 & 0.52 & 0.48 & 0.82 \\
\hline MAD & 1.7 & 1.2 & 2.4 & 0.91 & 0.51 & 1.1 & 0.34 & 0.36 & 0.64 \\
\hline \multicolumn{10}{|c|}{ low-[Mg/Fe] } \\
\hline median & 14.3 & 10.0 & 13.9 & 18.4 & 15.6 & 18.3 & 0.96 & 0.62 & 0.90 \\
\hline MAD & 3.9 & 2.5 & 2.7 & 3.8 & 3.3 & 3.5 & 0.50 & 0.30 & 0.27 \\
\hline \multicolumn{10}{|c|}{$\mathrm{I}-[\mathrm{Mg} / \mathrm{Fe}]$} \\
\hline median & 6.5 & 6.8 & 6.0 & 19.9 & 20.1 & 17.9 & 13.4 & 14.7 & 12.0 \\
\hline MAD & 0.9 & 0.78 & 0.73 & 0.98 & 0.38 & 0.43 & 2.6 & 1.5 & 1.9 \\
\hline
\end{tabular}



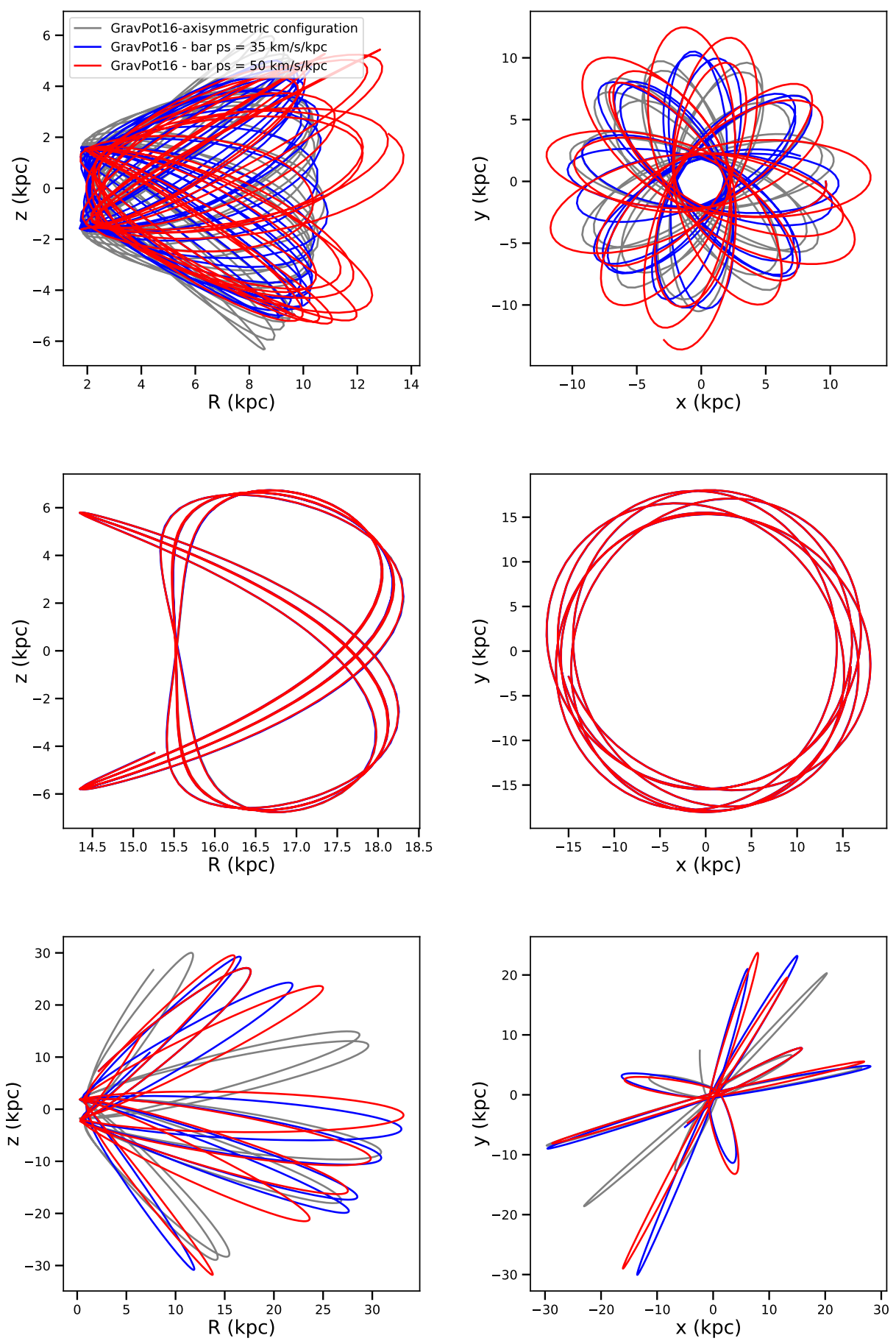

Figure 1. Computed orbits using an axisymmetric (grey) and non-axisymmetric configuration of GravPot16 potential model at different angular velocities for the bar (bar ps $=35 \mathrm{~km} \mathrm{~s}^{-1} \mathrm{kpc}^{-1}-$ red, and $50 \mathrm{~km} \mathrm{~s}^{-1} \mathrm{kpc}^{-1}$ - blue), for stars in our halo sample with [Fe/H] $>-0.75$. First row: A high- $[\mathrm{Mg} / \mathrm{Fe}]$ star, $\left\langle E>=-1449.064 x 10^{5} \mathrm{~km}^{2} \mathrm{~s}^{-2},<L_{z}>=70.304 x 10 \mathrm{kpc} \mathrm{km} \mathrm{s} \mathrm{s}^{-1},\left|z_{\max }\right|=5.546 \mathrm{kpc}\right.$, and $<e c c>=$ 0.79. Second row: An I- $\left.[\mathrm{Mg} / \mathrm{Fe}] \mathrm{star},\langle E\rangle>-1300 x 10^{5} \mathrm{~km}^{2} \mathrm{~s}^{-2},\left\langle L_{z}\right\rangle<-200 x 10 \mathrm{kpc} \mathrm{km} \mathrm{s} \mathrm{s}^{-1},<e c c\right\rangle<0.4$. Third row: A star with $E_{m}>-1000 \times 10^{5} \mathrm{~km}^{2} \mathrm{~s}^{-2}$ and $<L_{z}>>200 \times 10 \mathrm{kpc} \mathrm{km} \mathrm{s}^{-1}$. 